\title{
SINUHES NOTIZ ÜBER DIE KÖNIGE: SYRISCH-ANATOLISCHE HERRSCHERTITEL IN ÄGYPTISCHER ÜBERLIEFERUNG
}

Von Thomas Schneider

\section{§1 Einleitung}

Im Mittelpunkt der folgenden Studie ${ }^{1}$ steht ein bis heute änigmatisch gebliebener Passus in der klassischen mittelägyptischen Erzählung des Sinuhe. „A very difficult little section" urteilte A. H. GARDINER 1916, ${ }^{2}$ und am Ende des 20. Jahrhunderts zitierte W.K. Simpson die in ihm nach traditioneller Meinung genannten Herrscher als exemplarische Charaktere der literarischen Fiktion, deren historischer Nachweis nicht zu erwarten sei. ${ }^{3}$

Sowohl das Textverständnis an sich als auch die weitergehende historische Interpretation des Passus sind unklar. Der Abschnitt findet sich gegen Ende der Erzählung, die aller Wahrscheinlichkeit nach aus der Regierungszeit Sesostris' I. oder der Folgezeit stammen dürfte. ${ }^{4}$ Sinuhe, ein Leibwächter (ägypt. šmsw) König Amenemhats I., flieht nach der Ermordung Amenemhats I. aus Ägypten. Nach langer Odyssee durch syrisch-palästinische Territorien wird er von einem palästinischen Lokalfürsten aufgenommen, mit dessen Tochter vermählt, mit der Führung von dessen Armee betraut und steigt sel-

\footnotetext{
${ }^{1}$ Frühere Versionen dieser Ausführungen habe ich an der Universität München (6. 6. 2000), der University of Chicago (8. 1. 2001), der Universität Münster (15. 7. 2001) und der Universität Tübingen (29. 4. 2002) vorgetragen. Für Diskussionen danke ich insbesondere A. Gnirs und A. Loprieno.

2 A. H. Gardiner, Notes on the Story of Sinuhe, Paris 1916, 84.

3 „It is possible, although unlikely, that some of the characters in Sinuhe will come to light through texts in the Levant: the bedouin sheikh Amunenshi, the three governors loyal to Sesostris: ,Meki from Kedem, Khentiuwash from out of Keshu, and Menus, those who set your authority over the lands of the Fenkhu.' Similarly, some day an inscription of Tjeker-baal may be found near Beirut or that of a queen named Hatiba on Cyprus. Interesting and significant as these finds might be, they would not appreciably veer our understanding of the texts from fiction to reportage. Here again real events, such as those in Shakespeare's history plays, can be ,converted to literature." Vortrag am 8. ICE Kairo, April 2000, als Respondent von J. Baines. Ich danke W.K. Simpson herzlich für die bereitwillige Zurverfügungstellung seines Vortragstextes. Für eine möglicherweise mit Tjekerbaal zu verbindende Inschrift einer Pfeilspitze s. MOREnZ (wie Anm. 4), 7 Anm. 37.
}

ber zur Position eines lokalen Anführers auf. An diesem Punkt der Erzählung erfolgt ein Briefwechsel zwischen Sesostris I. und dem Exilierten, worauf Sinuhe nach Ägypten zurückkehrt, in Amt und Würde eingesetzt wird und ein Begräbnis erhält. Der Antwortbrief des Sinuhe an Sesostris I. - ein Schreiben, das ich nach der Terminologie der modernen Aktenkunde präziser als Immediatbericht, ${ }^{5}$ d.h. den unmittelbaren Bericht einer untergeordneten Person an den Landesherrn - bezeichnen möchte, hat in Pap. Berlin 3022 (Mittleres Reich; Sigel B) und (mit zahlreichen Abweichungen) dem Ostrakon Ashmolean Museum 1945.40 (Neues Reich; Sigel AOS) die fragliche Notiz überliefert. ${ }^{6}$

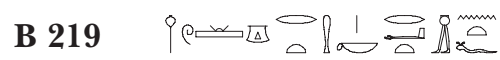

$$
\begin{aligned}
& \text { wc̣̆ MC̣̆3T grt ḥm=k rtj.t jnt=f } \\
& \text { Andin } \\
& \text { m-k-j F.S m qt-m HुsŚT }
\end{aligned}
$$

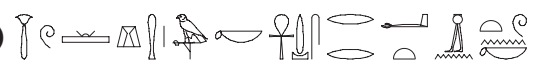

\footnotetext{
4 Dazu zuletzt C. Obsomer, Sinouhé l'Égyptien et les raisons de son exil, Le Muséon 112 (1999), 207-271 (17. Jahr Sesostris' I.). Eine Ansetzung unter Amenemhat II. favorisiert L. D. Morenz (nach mündlicher Mitteilung). Zur Textüberlieferung s. noch J. KAHL, „Es ist vom Anfang bis zum Ende so gekommen, wie es in der Schrift gefunden worden war". Zur Überlieferung der Erzählung des Sinuhe, in: M. Dietrich/I. KotTSIEPER (eds.), „Und Mose schrieb dieses Lied auf. “ Studien zum Alten Testament und zum Alten Orient. FS für Oswald Loretz, AOAT 250, Münster 1998, 383-400; L. D. Morenz, Kanaanäisches Lokalkolorit in der SinuheErzählung und die Vereinfachung des Urtextes, ZDPV 113 (1997), 1-18.

${ }^{5}$ H. O. MeISNER, Urkunden- und Aktenlehre der Neuzeit, Leipzig ${ }^{2} 1952,39$ (sub B. Schriftstücke der Unterordnung).

6 Textsynopse: R. Koch, Die Erzählung des Sinuhe, Bibliotheca Aegyptiaca XVII, Bruxelles 1990 (dort S. 66). Textzeuge B: A. H. Gardiner, Die Erzählung des Sinuhe und die Hirtengeschichte $=$ Hieratische Papyrus Berlin, Bd. 5, Berlin 1909, Tf. V-XV. Textzeuge AOS: J. W. B. BARns, The Ashmolean Ostracon of Sinuhe, London/ Oxford 1952.
} 


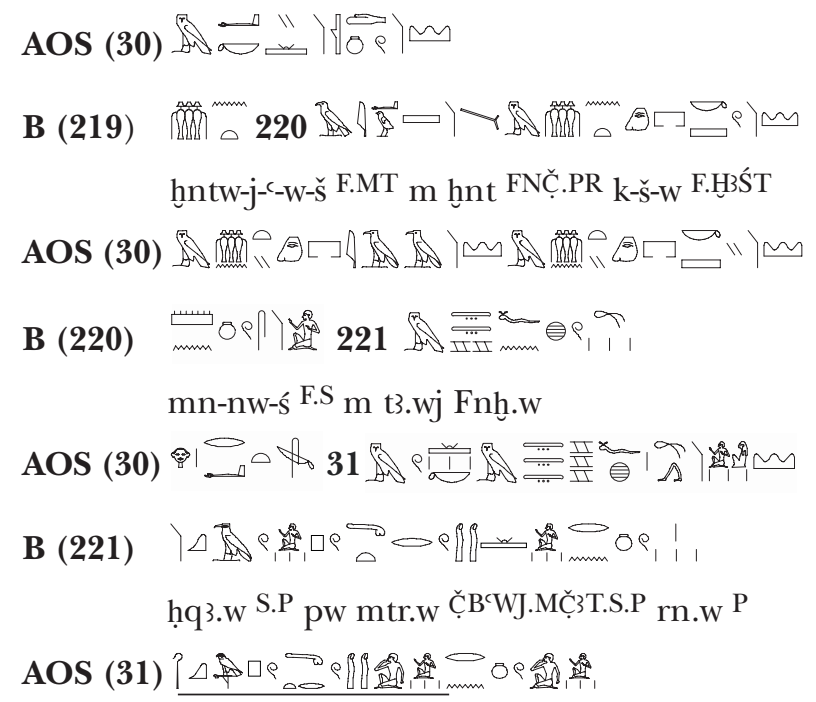

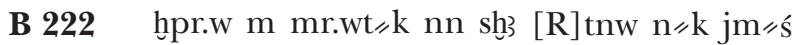
mjt.t čsm.w”k

Dieser Passus wurde seit Gardiner (1909) wie folgt verstanden und übersetzt:

[1] „Möge aber deine Majestät befehlen, daß man zurückbringe Meki aus Kedme, Khentiu-iausch aus dem Gefängnis Nubiens, und Menus aus den Ländern der Fenekhu; das sind Fürsten, gerecht an Namen (, die dich (stets) geliebt haben, ohne daß (man an sie) denkt. Retenu ist dein Besitz, ja (es ist) wie deine Hunde) “. ${ }^{7}$ (GARDINER 1909)

[2] „May thy Majesty command to cause to be brought Maki from Kedme, Khentiaush from Khentkesh, Menus from the lands of the Fenkhu. They are renowned princes (who have grown up in love of thee, albeit unremembered. Retenu is thine, like to thy hounds) " ${ }^{8}$ GARDINER (1916)

[3] „Möge aber deine Majestät befehlen, daß man Meki aus Kedemi und Chentiu-iausch aus Chent-Keschu und Menus aus den Ländern der Fenechu herbeihole; das sind Fürsten und ... Zeugen (, die in deiner Liebe aufgewachsen sind - ohne daß ich Retenu's gedenke: das ist dein, so wie es deine Hunde sind) “. 9 (ERMAN 1923)

[4] „Deine Majestät möge nun befehlen, daß man Maki aus Kedem und Chentiu-jausch aus dem Gefängnis

7 Gardiner, HPB V (wie Anm. 6), 13.

8 A. H. Gardiner, Notes on the Story of Sinuhe, Paris 1916 (hier sind auch die früheren Deutungen referiert), 84. Etwas anders $a a O$., 174: „Let now Thy Majesty cause to be brought".

9 A. ERMAN, Die Literatur der Aegypter. Gedichte, Erzählungen und Lehrbücher aus dem 3. und 2. Jahrtausend v.Chr., Leipzig 1923, 52.

10 G. RoEDER, Altägyptische Erzählungen und Märchen, Jena 1927, 35f.

11 G. LefEBVRe, Romans et contes égyptiens de l'époque pharaonique, Paris 1949, 19.
Nubiens herbeibringe und den Menus aus den syrischen Küstenländern. Sie sind Fürsten mit rechtschaffenem Namen (, die immer in Liebe zu dir verharrt haben, ohne daß man sich ihrer erinnert hat. Syrien gehört dir, es ist wie deine Hunde) ““. ${ }^{10}$ (Roeder 1927)

[5] „Que ta Majesté ordonne donc d'amener Méki de Qédem, Khentiouiâuch de Khentékéchou, Ménous du double pays des Fenkhou: ce sont des princes de bon renom (?) (, qui ont grandi dans ton amour. Inutile de mentionner le pays de Réténou: il est à toi, comme tes chiens) “. ${ }^{11}$ (LefEbvRe 1949)

[6] „Que ta Majesté ordonne de faire amener" les trois princes asiatiques "qui existent suivant tes volontés“. ${ }^{12}$ (Posener 1956)

[7] „And now your majesty decree to furnish for you an Eastern protector among the I'wš in (the region of) Ks and to give your guides (or services) in the distant lands: (saying) $\mathrm{O}$ righteous ruler, may porters exist upon your wish. Not shall Rethenu think: I am like one of your dogs“. ${ }^{13}$ (GOEDICKE 1965)

[8] „Let now thy Majesty command that there be brought unto thee the Meki man from Kedmi, the Foresters from out of Keshu, and the Menus man who spreadeth thine influence in the lands of the Fenkhu; they are princes of attested reputation, who have grown up in the love of thee; I will not make mention of Retjenu - it belongeth unto thee, even like as thine hounds“. ${ }^{14}$ (BARNs 1967)

[9] „May now your Majesty command that there be brought Meki from Kedem, / Khentiu-wash from out of Keshu, and Menus, those who set your authority over the lands of the Fenkhu. They are rulers whose names are worthy (and who have been brought up in your love. Not to mention Retenu, for it belongs to you even as your dogs) “. ${ }^{15}$ (SIMPSON 1972)

[10] „May then your Majesty command to have brought to you the prince of Meki from Qedem, the mountain chiefs from Keshu, and the prince of Menus from the lands of the Fenkhu. They are rulers of renown (who have grown up in the love of you. I do not mention Retenu - it belongs to you like your hounds". ${ }^{16}$ (LichtheIm 1973)

[11] „Moge Uwe Majesteit bevelen, dat hij meebrengt: Meki uit het Oostland, Chenti-awesj uit Chenti-koesj en Menoes uit de Beide Landen van Fenchoe. Dit zijn immers heersers met en gevestigde naam, die zich in uw

${ }^{12}$ G. Posener, Littérature et politique dans l'Égypte de la XII dynastie, Paris 1956, 113.

13 H. Goedicke, Sinuhe's Reply to the King's Letter, JEA 51 (1965), 29-47.

${ }^{14}$ J. Barns, Sinuhe's Message to the King: A Reply to a Recent Article, JEA 53 (1967), 6-14.

15 W.K. Simpson (Ed.), The Literature of Ancient Egypt. An Anthology of Stories, Instructions, and Poetry, New Haven/London 1972, 69.

16 M. LichtheIm, Ancient Egyptian Literature. A Book of Readings. Vol. I: The Old and Middle Kingdoms, Berkeley/Los Angeles/London 1973, 230. 
genegenheid koesteren. Zonder nog Retsjenoe te vermelden - want dat behoort $\mathrm{u}$ toe als uw jachthonden“. ${ }^{17}$ (BORGHOUTS 1974)

[12] „Auch möge deine Majestät befehlen, daß man veranlasse, den Meki zu holen aus dem Ostland, den ChentiJausch aus Chentikesch und den Menus aus dem Lande der Phönizier. Das sind Herrscher mit berühmten Namen (, die aus deiner Liebe hervorgegangen sind, ohne daß ich an Retenu denke, daß dir hörig ist wie deine Hunde) “. ${ }^{18}$ (SCHÜSSLER 1980)

[13] „Deine Majestät möge ferner befehlen, daß man Meki aus Kedem, Chentijawasch aus Chent-kuschu und Menus aus dem Land der Phönizier holen lasse. Es sind Herrscher mit gut beleumundeten Namen (, die in der Liebe zu dir aufgewachsen sind - nicht zu vergessen Retjenu, das dir gleichermaßen gehört, (dir ergeben) wie deine Hunde) “. ${ }^{19}$ (Blumenthal 1982)

[14] „Que Ta Majesté commande donc que luit soit amené Meki, originaire de Qedem, Khentiouiâoush, de Khentkeshou, et Menous du pays des Phéniciens; ce sont des princes au juste renom (et qui ont été élevés dans l'amour de toi. Je ne te rappellerai pas le pays du Retenou; il t'appartient, de même que (t'appartiennent) tes chiens)" ${ }^{20}$ (LALOuetTe 1987)

[15] „Ordini la Tua Maestà che le si portino il principe di Meki da Kedem, i capi dei paesi montuosi di Keshu e il principe di Menus, dalle due terre di Fenchu. Sono principi rinomati, (cresciuti nell'amore per te. Non ho bisogno di menzionare Retenu: esso ti appartiene come i tuoi cani) “..21 (BETRÒ 1990)

[16] „Deine Majestät möge aber befehlen, daß man holen lasse Meki aus Kedem, Chenti-jawasch aus Chent-kuschu, Menus aus dem Land der Phönizier. Es sind Herrscher mit gut beleumundeten Namen (, die in der Liebe zu dir aufgewachsen sind - nicht zu vergessen Retjenu: Dir gehört es gleichermaßen, wie Deine Hunde) “. ${ }^{22}$ (BLumenTHAL 1995)

[17] „Deine Majestät möge aber auch befehlen, daß man herbeihole den Meki aus dem Ostland, den Chentijawasch aus Chentkesch, den Menus aus dem Phönizierland - das sind Herrscher mit wohlbekanntem Namen, die sich durch deine Liebe entfaltet haben - ohne Retenus zu gedenken, das dir ergeben ist wie deine Hunde“. ${ }^{23}$ (Hornung 1996)

17 J. F. Borghouts, Egyptische Sagen en Verhalen, Bussum 1974, ${ }^{2} 1983,57$.

18 K. SCHÜssLER, Märchen und Erzählungen der Alten Ägypter, $1980,84$.

19 E. Blumenthal (Hg.), Altägyptische Reiseerzählungen. Die Lebensgeschichte des Sinuhe - Der Reisebericht des WenAmun, Leipzig 1982, 18.

20 C. Lalouette, Textes sacrés et textes profanes de l'ancienne Égypte. Mythes, contes et poésie, Paris 1987, 235.

21 M. C. BETRò, Racconti di viaggio e di avventura dell'antico Egitto, Brescia 1990, 53.

22 E. Blumenthal, Die Erzählung des Sinuhe, TUAT III: Weisheit, Mythen und Epen III, Gütersloh 1995, 904.

23 E. Hornung, Altägyptische Dichtung, Stuttgart 1996, $41 \mathrm{f}$.
[18] „Now, may your Majesty command that he be made to bring the Meki man from Qedem, the settler from out of Keshu, and the Menus man from the lands of the Fenkhu. They are rulers who are well known (, who live by love of you. Without calling Retjenu to mind - it is yours, even like your hounds!) “. ${ }^{24}$ (PARKINSON 1997)

[19] „Que tu majestad ordene que te sea traído Meki de Qedem, en Iraru, y de KeSu, Menus, realizando (así) tus designios en las tierras de los Fenju. Ellos son gobernantes de renombre (, que han crecido queriéndote; sin mencionar Retenu, que te pertenece como si fueran tus perros) “. ${ }^{25}$ (GALÁn 1998)

[20] „So, let your Majesty command that this your servant bring you back the prince of Qedem, the chieftain of the Iawesh, south of Keshu, and the leader of the two Phoenician coastlands“. ${ }^{26}$ (FOSTER 2001)

Dieser Überblick illustriert in wünschenswerter Deutlichkeit die Problematik:

$>$ Ist die Rede von (drei individuellen?) Herrschern aus dem Raum Palästina oder von Toponymen?

> Falls einzelne Herrscher gemeint sind: werden sie nach Ägypten gebracht - als Freunde, ${ }^{27}$ Geiseln ${ }^{28}$ oder begnadigte Verbannte ${ }^{29}$ - oder dem ägyptischen König lediglich anempfohlen? ${ }^{30}$

$>$ Oder ist die Rede von Gebieten, die dem ägyptischen König loyal sind ${ }^{31}$, die von Sinuhe für den ägyptischen König gewonnen wurden ${ }^{32}$ oder mit denen Sesostris I. Kontakt knüpfen soll ?

Die folgenden Überlegungen sollen diese Fragen einer Lösung zuführen.

\section{\$2 KRITIK ZWEIER DEUTUNGEN \\ (M. GreEN - 1983; K. A. KitCheN - 1994)}

In jüngerer Zeit sind zwei Versuche unternommen worden, den Herrschervermerk der Erzählung zu entschlüsseln. Am einflußreichsten war der Ansatz M. GreEns von 1983, die mit dem Determinativ der Person bzw. des gefallenen Feindes versehenen Begriffe

24 R. B. PARkinson, The Tale of Sinuhe and other Ancient Egyptian Poems 1940-1640 BC, Oxford 1997, 38.

25 J. M. Galán, Cuatro Viajes en la Literatura del Antiguo Egipto, Madrid 1998, 92.

26 Ancient Egyptian Literature. An Anthology, translated by J. L. Foster, Austin 2001, 142.

27 Kitchen (wie Anm. 38).

28 GARdiner (wie Anm. 8), 84.

29 Gardiner (wie Anm. 6), 13 Anm. 7.

30 „These three foreign rulers are thus commended by Sinuhe to the king“" (SIMPSON, wie Anm. 15, 68 n. 17).

31 Blumenthal (wie Anm. 22), 904 Anm. in Anlehnung an Green (wie Anm. 33).

32 Parkinson (wie Anm. 24), 50 n. 59. 
in B 219-221 nicht als Personennamen, sondern trotz der Determinierung als Bezeichnungen von Gebieten zu verstehen: ${ }^{33}$ „Amki in Qedem; the inhabitants of the cedar forest (= Lebanese) in Geshur; Amanus in the land of the Fenchu/carpenters“. Dabei identifiziert er $m k j$ mit Amki (= semit. 'amq "Talebene“), der südlichen Beqā̄ bzw. dem mittleren Orontestal, qtm mit semit. qdm „Osten“, möglicherweise einem Gebiet östlich des Libanon; kšw mit Geschur, der Region des Golan/Berges Hermon, und mnnws mit dem nordsyrischen Gebirgszug des Amanus. Dieser Ansatz wurde von zahlreichen Forschern (u.a. C. EDER $^{34}$ ) übernommen, meist insofern modifiziert, als man Nisbe-Ableitungen („der von Meki“, „der von Menus“) postulierte, um der Notation (Personendeterminative) Genüge zu tun. Entsprechen werden die Vermerke zu „il principe di Meki“, „il principe di Menus“ (so die zitierte Übersetzung von BETRò 1990) bzw. „the Meki man“, „the Menus man“ (so die zitierte Übersetzung von PARKINSON 1997) ergänzt. Eine derartige Restitutionsmöglichkeit wird vom Textbefund aber nicht gestützt (die dafür bemühten Belege notieren stets die Nisbe-Ableitung ${ }^{35}$ ); außerdem wäre ein einfaches Ethnikon als referentielle Benennung eines Herrschers merkwürdig. Davon abgesehen ist die Deutung Greens aber aus einer ganzen Anzahl von Gründen ausgeschlossen:

1. Die Notation differenziert deutlich zwischen der dreimaligen Angabe von Personen und ihrer Zuweisung zu Toponymen. Der Vermutung Greens (Irrtum des Schreibers) ist a priori eine Erklärung vorzuziehen, die die personalen Determinative des Textes zugrunde legt.

2. Bei einer Gleichung $m k j=$ Amki/'amq ist der Pharyngal 'Ajin nicht notiert bzw. die ganze erste Silbe apokopiert.

3. semitisches /q/ (camq) wird nie durch ägypt. <k> wiedergegeben.

33 M. GReEn, The Syrian and Lebanese Topographical Data in the Story of Sinuhe, CdE 58 (1983), 38-59.

34 C. EDER, Die ägyptischen Motive in der Glyptik des östlichen Mittelmeerraumes zu Anfang des 2. Jts. v.Chr., OLA 71, Leuven 1996, 173.

35 Barns (wie Anm. 6), 23f.; E. Edel, in: O. Firchow (Hg.), Ägyptologische Studien. Hermann Grapow zum 70. Geburtstag gewidmet, Berlin 1955, 566.

36 Siehe W. Helck, Zur Verwaltung des Mittleren und Neuen Reiches, PÄ 3, Leiden 1958, 419; ders., Priester, Priesterorganisation, Priestertitel, LÄ IV, 1086; P. Posener-KRIÉGER, Les archives du temple funéraire de Neferirkarê-Kakai (les papyrus d'Abousir). Traduction et commentaire, 2 vols, BdE LXV/1-2, Le Caire 1976, Reg. p.659; W.A. WARD,
4. Der von Green postulierte Ausdruck *hntw-`̌̌ „Zedernwaldbewohner" existiert in dieser Konsonantengestalt nicht; er kann auch phonologisch nicht dem schon im Alten Reich belegten ägyptischen Ausdruck ḩntw-š zugrunde gelegen haben (wie von Green suggeriert), der jedenfalls eine andere Bedeutung besitzt, auch wenn er weiterhin Gegenstand der Diskussion ist. ${ }^{36}$ Ein hypothetisches ‘š „Zeder“ könnte schließlich nicht (wie im vorliegenden Text) $<j^{\mathrm{c}} w \check{s}>$ geschrieben werden.

5. Bei einer Gleichung $k \breve{w}=$ hebr. gěsûr muß das fehlende auslautende $-r$ erklärt werden.

6. Die Gleichung mnnwś = Amanus setzt wiederum eine Apokopierung der Anlautsilbe voraus. Verunmöglicht wird sie dadurch, daß -us erst die lateinische Endung ist (entsprechend mit griechischer Endung Amanos), während die akkadische Form Amanu lautete. Dies trotz Greens Spekulation: „It may reasonably be inferred that the $s$ of Amanus is a classical affix, as it is not present in any of the cuneiform variants of the toponym. At the same time the lack of a final $s$ in the cuneiform spellings does not mean conclusively that the local Anatolian spelling had no such $s$. It seems pointless to comment further on this matter here as, irrespective of the standpoint adopted, the remaining data seems strong enough to support the identification proposed here". 37

Nach M. Green hat m.W. nur noch K. A. KITCHEN (1994) einen Lösungsvorschlag unterbreitet. ${ }^{38}$ Er lehnt völlig zu Recht die Deutung der mit Personaldeterminativ versehenen Ausdrücke als Namen von Gebieten ab und schlägt vor: „Meki [Personenname] from Qedem [semit. ,Osten“; östlich des Libanon]; the leader Yacush [ein Personenname: ,(der Gott NN) wird helfen') from Kushan (in Edom), Mennus(i) (,he from Mennus [= Minos]' = ,the Minoan') from the (two) lands of the Fenkhu

Index of Egyptian Administrative and Religious Titles of the Middle Kingdom, Beirut 1982, 40 [304.]; A. M. Roth, Egyptian Phyles in the Old Kingdom. The Evolution of a System of Social Organization, SAOC 48, Chicago/Illinois 1991, 81; dies., The Distribution of the Old Kingdom Title Hntj-šs, in: Akten des vierten Internationalen Ägyptologen-Kongresses München 1985, Bd. 4, Hamburg 1991, $177-185$.

37 Green (wie Anm. 33), 53.

38 K. A. Kitchen, Sinuhe's Foreign Friends, and Papyri (Coptic) Greenhill 1-4, in: C. Eyre, A. LeAhy, L. MonTAgno Leahy (eds.), The Unbroken Reed. Studies in the Culture and Heritage of Ancient Egypt in Honour of A. F. Shore, London 1994, 161-169. 
(= Crete and Phenicia)“. Diesem Ansatz steht entgegen:

1.Der Text hat nicht den Sg. hntj („leader“), sondern notiert (hieratisch ganz eindeutig) den Pl. hntw.

2. Der Personenname kann nicht als analog zu hebr. jacuš „(ein Gott NN) wird helfen“ verstanden werden, da die entsprechende semitische Wurzel * $\dot{\mathrm{g} w t}$ lautet (ägyptisch wäre für den Imperfektnamen dann etwa <ygwś $>$ zu erwarten). ${ }^{39}$

3.Die Schlußsilbe -ān von Kušān wäre nicht notiert.

4. $<$ mnnwś $>$ ist keine Nisbebildung und kretisch ,Minos` kein Ortsname;

5.Die Endung -os von ,Minos“ ist aller Wahrscheinlichkeit nach griechisch;

6. Das Gebiet der Fenchu umfaßt nicht Kreta.

Diese Argumente schließen auch den Ansatz Kitchens aus.

\section{$\S 3$ Ein Neuansatz}

Der Neuansatz, den ich im Folgenden vorschlage, legt folgende Eckpunkte zugrunde:

1. Die Rahmung des Herrschervermerks spricht in B 222 von den umstrittenen Ausdrücken als rn.w. Damit sind keine Namen (und in Sonderheit keine Personennamen) gemeint. Ägyptisch $r n$ bedeutet ebenso „Name einer Sache, Titel, Bezeichnung“; $r n . w$ werden etwa auch die Titulaturnamen des ägyptischen Königs genannt. Was der Passus in Tat und Wahrheit anführt, sind nicht Eigennamen, sondern offizielle Herrschertitel, und die Territorien, über die diese Herrscher regierten. Jeder dieser Titel ist mit determiniert, was einen fremden Ursprung des Titels anzeigt.

2. In der Passage ist nicht die Rede von Fürsten Palästinas. Der Text selber stellt fest, daß Palästina hier nicht angesprochen wird, da es ja dem ägyptischen König ohnehin schon wie Hunde treu ergeben ist. Stattdessen konzentriert sich die Perspektive hier auf Nordsyrien und Südostanatolien.

3. Die erwähnten Titel und Toponyme spiegeln die komplexe linguistische Situation wieder, die zu Beginn des 2. Jahrtausends für den genannten Bereich vermutet werden kann (neben Semitisch auch Luwisch und Hurritisch).

\footnotetext{
39 Alternativ verweist R. ZADOK, The Pre-Hellenistic Israelite Anthroponymy and Prosopography, OLA 28, Leuven 1988, 24 auf die Wurzel 'wš (?) „sich kümmern um“.

40 H. Goedicke, Where Did Sinuhe Stay in Asia (Sinuhe B 29-31)? CdE 67(1992), 28-40.
}

Die folgende Diskussion präsentiert zunächst die einzelnen Informationen mit den HerrscherPrädikationen und den Angaben der Territorien, die ich als 1.-3. Vermerk bezeichnen werde, dann den Rahmentext.

\section{§3.1 Der erste Vermerk}

Der erste Vermerk umfaßt eine Personenbezeichnung $\rightarrow$ A / $\mathrm{m}$-k-j F.S und eine adverbielle Angabe

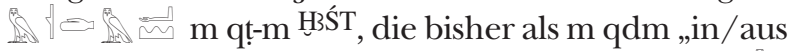
Qedem“ aufgefaßt wurde (auslautend auf $=$ $<m>$ [Gardiner M20], nicht $\left.\langle m\rangle+<^{\text {' }}\right\rangle$ ); einzig H. GOEDICKE plädierte für eine abweichende Lesung als $<\mathrm{j}$-ṭ-m> „Edom“. ${ }^{40}$ Die überwiegend vollzogene Identifizierung mit semit. $q d m$ „Osten“ ist aus sachlichen und phonologischen Gründen kaum plausibel. Phonologisch wäre für die Entstehungszeit des Sinuhe die Wiedergabe von semit. /d/ durch ägypt. /t / (ägyptologisch $<\mathrm{d}>$ ist unkorrekte Konvention) erklärungsbedürftig. Da der folgende zweite Vermerk in B 220 einen Beleg für die Vermutung liefert, wonach der genuine Lautwert / d/ des konventionell $<$ '> notierten ägyptischen Phonems in der Tat noch bis ins frühe Mittlere Reich vorlag, wird die Annahme einer differierenden Wiedergabe von /d/ in B 219 noch weniger plausibel. Sachlich ist gegen die Ansetzung des semitischen Ausdrucks einzuwenden, daß qdm m.W. im Semitischen des 2. vorchristlichen Jahrtausends nur als Richtungsangabe verwendet wird, aber nie als Ortsbezeichnung im Sinne von „Ostland“. ${ }^{40 a}$ Bei einer gewöhnlichen direktionalen Präzisierung („im Osten [eines bestimmten Gebietes]“) hätte der ägyptische Verfasser aber sicher ägyptisch $j 3 b t . t$ verwendet.

Eine überzeugende Lösung ergibt sich bei Betrachtung der Bezeugungen dieses Toponyms in den einzelnen Handschriften. Die älteste (Berliner) Handschrift zeigt nämlich die Variantenschreibung

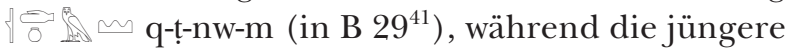
Version des Ashmolean Ostracon aus dem Neuen Reich in der Überlieferung der Stellen B 182/B

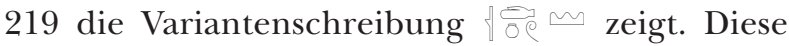
Notationen stellen dann aber offenbar perfekte Wiedergaben des Ortsnamens Qațanum (ältere Form mit Mimation) bzw. Qața (jüngere Form nach Abfall der Mimation) dar. Da das ägyptische

\footnotetext{
40a Dazu noch S. Mittmann, ZDPV 118 (2002), 50f., Anm. 44.

41 Diese Notation stützt außerdem die obige Ansetzung von als $<\mathrm{m}>$ [Gardiner M20], nicht $<\mathrm{m}>+$ $<$ '>
} 
Wort $\{\odot$ qt „rund sein“ seit der 11. Dynastie zunehmend mit nw-Topf statt Kreis ( $(\vec{\circ})$ geschrieben wurde, erklären sich die Notationen ohne nw-Topf in der Art von $\_-\infty$ als Hyperkorrektur (Fortlassung eines als ägyptisches Determinativ mißverstandenen eigentlichen Phonogramms).

$\mathrm{Zu}$ Beginn des 2. Jahrtausends v. Chr. ist das Reich von Qatanum mit seiner Hauptstadt am mittleren Lauf des Orontes (heute Tell el-Mišrife) die vorherrschende politische Macht in Zentralsyrien. ${ }^{42}$ Diese Identifizierung paßt genauestens zu der Beschreibung, die Sinuhe selber in B 29 über seine Reiseroute gibt: „Nachdem ich mich von Byblos gelöst hatte, näherte ich mich [wie wir jetzt lesen:] Qațanum (fh.n”j r kpnj ḥsj.n`j r Qṭnwm)“, von wo ihn sein nachmaliger Oberherr 'mmwnnšj nach Nordpalästina (Rčnw hrj) holte. Sie stimmt genau mit dem Verlauf der Überlandwege des frühen 2. Jahrtausends überein: von Byblos nordöstlich über den Libanon nach Qațanum, von dort jedoch auf der Binnenroute durch die Beqā̄ südwärts bis nach Nordpalästina (Abb. 1) $:^{43}$

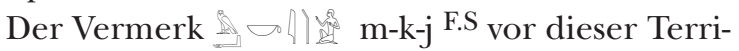
torialangabe kann als Herrschertitel erklärt werden. Auf der m.E. richtigen Spur waren schon K.A. Kitchen $^{44}$ und G. Scandone Matthiae, ${ }^{45}$ die beide auf den eblaitischen König Mēki/Mēgi verwiesen, der als Protagonist des hurritisch-hethitischen Epos von der Freilassung erscheint. Mit dessen Name sei der angebliche Personenname des Sinuhe-Vermerks vergleichbar (KITCHEN) bzw. der in dem vorliegenden Sinuhe-Vermerk genannte Meki sei mit dem für Ebla bezeugten möglicherweise identisch (ScANDOnE MATthiAe). Für unseren Zweck ausschlaggebend ist der Umstand, daß es sich bei dem genannten Beleg vermutlich um die verunstaltete Form einer Bezeichnung handelt, die in zeitgenössischen nordsyrischen Quellen des 20.-18. Jahrhunderts v. Chr. belegt ist, und bei der es sich vermutlich um ein Epitheton oder einen Herrschertitel handelt. Zuletzt hat C. KüHNE ${ }^{46}$ den im Sinuhe überlieferten Ausdruck $m k j$ zu diesen Belegen in Verbindung gesetzt:

1. Mēki/Mēgi von Ebla im hurritisch-hethitischen Epos von der Freilassung. ${ }^{47}$ Der auch als „Stern von Ebla“ bezeichnete Mēki/Mēgi wird als Oberherr von neun Klientelkönigen dargestellt. E. NEU plädierte für eine Ansetzung des Mēgi, mMe-e-ki, Nom. mMe-e-ki-iš notierten Namens als /Mēgi/ ${ }^{48}$ und vermutete: „Auch wenn innerhalb der Bilingue Mēgi mit Personendeterminativ geschrieben wird, könnte diese Namensform ursprünglich ein Epitheton oder auch ein Herrschertitel der Könige von Ebla gewesen sein - mit sekundärer
${ }^{42}$ H. KLengel, Geschichte Syriens im 2. Jahrtausend v.u.Z., Teil 2: Mittel- und Südsyrien, Berlin 1969, 96-138; ders., Syria 3000 to 300 B.C.: a Handbook of Political History, Berlin 1992, 65-70; F. ZeEB, Die geographischen Bezeichnungen aus Alalah VII, UF 30 (1998), 1999, 829-886: 847; G. F. Del MonTe, J. Tischler, Die Orts- und Gewässernamen der hethitischen Texte (RGTC 6 = BTAVO Reihe B. 7, 6), Wiesbaden 1978, 197.203; M. BONECHI, I nomi geografici dei testi di Ebla (RGTC 12/1, = BTAVO Reihe B. 7, 12.1), Wiesbaden 1993, 139.162f.

${ }^{43}$ F. JOANNÈs, Routes et voies de communication dans les archives de Mari, in: J.-M. Durand (ed.), Mari, Ebla et les Hourrites: dix ans de travaux. Actes du colloque international (Paris, mai 1993), Paris 1996, 323-361: Karte S. 325.

44 K. A. Kitchen, wei Anm. 38, 162.

45 G. Scandone-Matthiae, Méki/Mekim (d'Ebla) dans l',histoire de Sinouhé“?, MARI 8 (1997), $249 \mathrm{f}$.

46 C. Kühne, Meki, Megum und Mekum/Mekim, in: S. ISRE'El, I. Singer, R. ZADOK, Past Links: Studies in the languages and cultures of the ancient Near East, Winona Lake 1998, IOS 18, 311-322 (die Kenntnis dieses Aufsatzes verdanke ich L. Morenz).

47 E. Neu, Das hurritische Epos der Freilassung I. Untersuchungen zu einem hurritisch-hethitischen Textensemble aus
Hattuša, Studien zu den Boğazköy-Texten, H. 32, Wiesbaden 1996, 282, 443f., 479ff.; Rez. von D. Prechel, ZDMG 148 (1998), 385-390; V. HAAS, I. WEGNER, Literarische und grammatikalische Betrachtungen zu einer hurritischen Dichtung, OLZ 92 (1997), 438-455; dies., IstMitt 43 (1993), 53-58. S. u.a. noch H. OtтEN, Ebla in der hurritisch-hethitischen Bilingue aus Boğazköy, in: Wirtschaft und Gesellschaft von Ebla, Heidelberger Studien zum Alten Orient 2, 1988, 291f.; E. NeU, Mehrsprachigkeit im Alten Orient - Bilinguale Texte als besondere Form sprachlicher Kommunikation, in: G. BINDER, K. EHLICH (Hg.), Kommunikation durch Zeichen und Wort. Stätten und Formen der Kommunikation im Altertum IV, Trier 1995, 11-39 (24f.: Lit. zur Bilingue); ders., IstMitt 43 (1993), 59ff.; H. A. HoffNer, Jr., Hurrian Civilization from a Hittite Perspective, in: G. Buccellati, M. Kelly-Buccellati (eds.), Urkesh and the Hurrians. Studies in Honor of Lloyd Cotsen, Bibliotheca Mesopotamica 26 = Urkesh/Mozan Studies 3, Malibu 1998, 167-200: 182f.; KüHNE (wie Anm. 46), 312.

48 E. Neu, Das hurritische Epos der Freilassung I (wie Anm. 47), 406f., 499. 


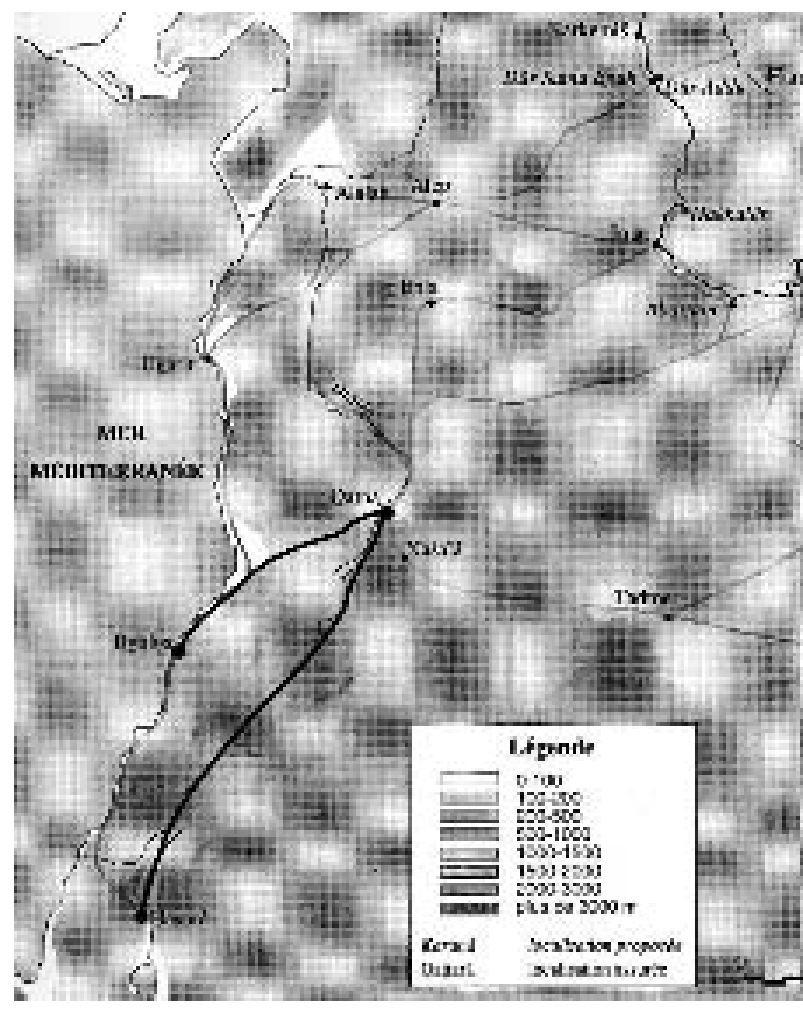

Abb. 1 Verlauf der Routen Byblos - Qatna - Hașura

Umdeutung zum Personennamen hier in der Erzählung um die Stadt Ebla.“

2. Ein ensivon Ebla Megum in einer neusumerischen Rationenliste aus dem 7. Jahr des Amar-Sin (3. Dyn. von Ur): Me-gu-um énsi Eb-laki ${ }^{49}$

3. Die Selbstbezeichnung des Herrschers als me-ki-im eb-la-i-im in der Votivinschrift der Statue des IbbitLim aus Ebla. ${ }^{50}$ Hier ist der Ausdruck sicher als Titel zu bewerten.

4. Der Brief Mari A.877 des Yatarum an Aplahanda von Karkemisch erwähnt einen König Mekim von Apišal. ${ }^{51}$

49 D. I. Owen, R. Veenker, MeGum, The First Ur III Ensi of Ebla, in: L. CAGNI (ed.), Ebla 1975-1985, Napoli 1987, 263ff.; G. Wilhelm, Art. Meki, Meg/kum, RA 8, 1993-7, 33; H. Klengel, Syria 3000 to 300 B.C., Berlin 1992, 36, 64 Anm. 114; KüHNe (wie Anm. 46), $315 f$.

50 D. I. OwEn, $a a O$., 271f. (identifiziert mit ihm den Megum [2.]). Zu Ibbit-Lim: G. Pettinato, Inscription de Ibbit-Lim, Roi de Ebla, AAAS 20 (1970), 19ff.; M. V. Tonietti, D. Charpin, N. Ziegler, G. ScandoneMatthiae, MARI 8 (1995), 225ff.; G. Wilhelm, Art. Meki, Meg/kum, RA 8 (1993-7), 33; KüHNE (wie Anm. 46), $314 \mathrm{f}$.

51 G. Wilhelm, Art. Meki, Meg/kum, RA 8 (1993-7), 33; D.
5. Zwei Abrollen von Siegeln aus Kültepe II, 527 und $529 \mathrm{a}$, die als casus rectus mekum belegen. ${ }^{52}$

Die jüngste Deutung ${ }^{53}$ dieses möglichen altsyrischen Königstitels wurde von M. Bonechi ${ }^{54}$ und ausführlicher M.-V. Tonietti ${ }^{55}$ erwogen. Sie sehen in dem Titel eine dialektale Nebenform zu mlk „König“, vergleichbar dem Gottesnamen Hēbat (< Halbat „Die von Aleppo“), wobei die als mēkim belegten Formen allerdings als casus obliquus erklärt werden müssen. Dieser Deutung hat sich auch C. Kühne angeschlossen und den im Sinuhe genannten $M k j$ ebenfalls zu den altsyrischen Belegen des Titels gestellt. ${ }^{56}$ Aber auch bei Ablehnung der Ableitung von malkum und Ansetzung eines genuinen /g/ kann die ägyptische Notation zu dieser Bezeichnung gestellt werden, da semit. /g/ regulär auch durch ägypt. $\langle k\rangle$ transkribiert wird. Die Schreibung in Sinuhe B 219 dürfte damit als Wiedergabe eines syrischen Königstitels mēkim (mēkum ?) zu verstehen sein; f entspricht als Vokalandeutung für /i/ im In- oder Auslaut dem Umschriftsystem des Mittleren Reiches; das auslautende $<\mathrm{m}>$ wäre vor der nachfolgenden Präposition $<\mathrm{m}>$ nicht notiert (Haplographie). Vermerk 1 der Herrschernotiz ist daher wie folgt aufzufassen:

„Der Mēkim (semitischer Königstitel; < malkim/ malkum ?) in Qațanum“.

\section{\$3.2 Der zweite Vermerk}

Der zweite Vermerk der Notiz irritierte bisher am meisten. Er ist äußerlich gleich wie der erste und dritte Vermerk als Bezeichnung einer Person Q A bisherigen Lesung) aufgebaut. Dieser Ausdruck setzt auf den ersten Blick mit einem ägyptischen Nomen hnt.w „die Vorderen, Vorsteher“ ein; eine

Charpin, N. Ziegler: Mekum, roi d'Apišal, MARI 8 (1997), 243-258; KüHNe (wie Anm. 46), 230f.

${ }_{52}$ M.-V. Tonietti (wie Anm. 55), 226-230; KüHne (wie Anm. 46), 314f.

53 Die ältere Diskussion ausführlich referiert bei TONIETTI (s. Anm. 55) und KÜHNE (s. Anm. 46).

54 M. Bonechi, II Millennium Ebla Kings, RA 91 (1997), 33-38: S. 35.

${ }^{55}$ M.-V. Tonietri, Le cas de Mekum: continuité ou innovation dans la tradition éblaite entre $\mathrm{III}^{\mathrm{e}}$ et $\mathrm{II}^{\mathrm{e}}$ millénaire? MARI 8 (1997), 225-242 (hier auch Diskussion aller zuvor genannten Belege).

${ }^{56}$ KüHne (wie Anm. 46), Nachsatz S. 31. 
ägyptische Lesung des Ausdrucks scheiterte jedoch in der Vergangenheit an den nachfolgenden Hieroglyphen vor der Determinierung. Ich vermute, daß in Tat und Wahrheit eine Wortschreibung vorliegt, d.h. das bekannte ägyptische Verfahren, zur Umschrift eines fremdsprachigen Wortes ein ähnlich lautendes ägyptisches Wort zu verwenden. Die zeitgenössische Lautung des Wortes hnt.w kann als *hánt ${ }^{\circ}$ rekonstruiert werden. ${ }^{57}$ Die folgende Schilfblatthieroglyphe steht bei Umschriften des Mittleren Reiches in Anfangsposition (wie hier nach der Wortschreibung *hantaw) für den Wert $/{ }^{2} \mathrm{a} /$ (s. aber unten zur Reihenfolge der Zeichen). Entscheidend ist die phonologische Interpretation der nachfolgenden Arm-Hieroglyphe für die vorliegende Zeit des frühen Mittleren Reiches. Während dieses Phonogramm seit dem hohen Mittleren Reich zur Wiedergabe von semitischem 'Ajin verwendet wird, entspricht das von ihm repräsentierte Phonem genuin semitischem Dalet. ${ }^{58}$ Neuerdings konnte gezeigt werden, daß dieser Lautwert auch noch für den Beginn des Mittleren Reiches anzusetzen ist. ${ }^{59}$ Dies vorausgesetzt, ergibt sich für die Bezeichnung bis hierher die ungefähre Lautung */hantaw-a-d/. Diese Lautfolge kann aber schwerlich etwas anderes wiedergeben als das luwische Lexem hantaunatti"Herrscher" (mit Wiedergabe von luwischer Tenuis bzw. Fortis [t-d für t-tt]). Für luwisch hantaunatti- und die direkt verwandten Bildungen jüngerer anatolischer Sprachen vgl.:

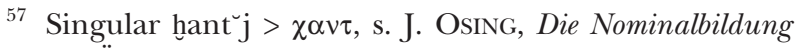
des Ägyptischen, 2 Bde, Mainz 1976, 315 mit Anm. 1347. Zur Lesung und Vokalisierung von $t w$ (nicht tjw) s. E. EDEL, Neue Deutungen keilschriftlicher Umschreibungen ägyptischer Wörter und Personennamen, (Phil.-hist. Kl. der ÖAW, Sitzungsberichte, 375. Bd.), Wien 1980, $47 \mathrm{f}$.

58 O. Rössler, Das Ägyptische als semitische Sprache, in: Schriften zur Semitohamitistik, hg. von T. SCHNEIDER unter Mitarbeit von O. KaELIN, AOAT 278, Münster 2001, 543-606; T. SCHNEIDER, Beiträge zur sogenannten „neueren Komparatistik“, LingAeg 5 (1997), 189-209; F. KAMMERZELL, Zur Umschreibung und Lautung, in: R. Hannig, Die Sprache der Pharaonen. Großes Handwörterbuch Ägyptisch-Deutsch (2800-950 v.Chr.), Mainz 1995, XLII-LI: S. XLVI.L; W. SCHENKEL, Einführung in die ägyptische Sprachwissenschaft, Darmstadt 1990, 50; C. Peust, Egyptian Phonology. Introduction to the Phonology of a Dead Language, Göttingen 1999, 106; A. LOPRIENO, Ancient Egyptian. A Linguistic Introduction, Cambridge 1995, 31.45; J. P. Allen, Middle Egyptian. An Introduction to the Language and Culture of Hieroglyphs, Cambridge 2000, 15.

${ }^{59}$ F. KAMmERZELL, Zur Interpretation einiger Beispiele graphemsprachlicher Varianz im Ägyptischen, Göttinger

\section{1. keilschrift-luwisch}

Keilschrift-luwisch hantaunatti-, bezeugt in KUB XXXV 123 (13. Jh.). Dazu urteilte F. Starke: „Das nominale Grundwort, hantauxatti- c., ist ebenfalls nur in diesem Text bezeugt und vermutlich auf Pirua zu beziehen: Sg.N. ha-an-da-uxa-te-eš, A. ha-an-da-uxa-teen ibid. IV? 1', 12' (vgl. StBoT 30, 251). Zur Einfachschreibung des Dentals im Suffix -tti- ist festzustellen, daß die junge Niederschrift auch sonst die graphische Darstellung der Fortis vernachlässigt. Über die Bedeutung läßt sich dem fragmentarischen Text nichts Sicheres entnehmen. Einen Anhaltspunkt dürfte jedoch das verwandte (wenngleich im Suffix

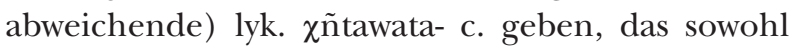
Titel von Göttern als auch von hochgestellten, politisch einflußreichen Persönlichkeiten ist und im Griechischen mit $\beta \alpha \sigma i \lambda \varepsilon v$ s übersetzt wird. Neben dem Titel steht ein in Datierungsformeln vorkom-

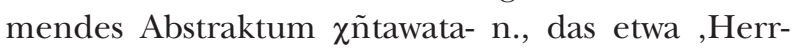
schaft, Regierung' bedeutet und funktional dem k.luw. hantauxattāhit- entsprechen könnte. Beide lyk. Nomina sind von $\chi$ ñtewe- (TL 26,2: $\chi$ ñtawa-) abgeleitet, das H. Pedersen, LuH 17 als ,führen' gedeutet hat; vom gleichen Stamm ist sicher auch das k.luw. hantauxatti - gebildet ${ }^{*}{ }^{60}$ H. C. MelCherT übersetzt „supreme authority, king“. ${ }^{61}$

\section{2. hieroglyphen-luwisch: REX-ti (fraglich)}

E. LAROCHE vermutete, daß „faute de lecture phonétique complète, on ne peut affirmer l'identité de

Beiträge zur Sprachwissenschaft 2 (1999), 61-97: 61-78; vgl. für das ausgehende Alte Reich T. SCHNEIDER, Wer war der Gott „Chajtau“?, in: K. M. CiaŁowicz, J. A. OstROWski (Hgg.), Les civilisations du Bassin Méditerranéen. Hommages à Joachim Sliwa, Cracovie 2000, 215-220.

${ }^{60}$ F. STARKe, Untersuchung zur Stammbildung des keilschriftluwischen Nomens, StBoT 31, Wiesbaden 1990, 171, bei der Diskussion des Nomens hantauxattāhit-n. „Amt(sbereich) eines hantaunatti-“.

61 H. C. Melchert, Cuneiform Luvian Lexicon, Chapel Hill 1993, 52: handawat(i)- ,supreme authority, king“, NSg ha-an-da-wa-te-eš , position of supreme authority, kingship“ N-ASg ha-an-ta-wa-da-ḩi-ša; E. LAROCHE, Dictionnaire de la langue louvite, Paris 1959, 40. Noch A. Heubeck, StMed 1 (1979 [80]), 247ff., vgl. auch ders., Acta Philologica Aenipontana 4 (hrsg. von R. MUTH), 1979, 51f.; A. BERnabé, The Luwian Abstracts in -ahi(t) and the Hittite Nouns in -ai-. A Critical Review, in: YoEL L. Arbeitman (ed.), A Linguistic Happening in Memory of Ben Schwartz. Studies in Anatolian, Italic, and other IndoEuropean Languages, Louvain-la-Neuve 1988, 107-129, S. 108 (luw. h.andawati- „leader (?) “; abstr. („case“ in -ša) hantawatahiša „leadership (?) “, corresponding to Lyc. $\chi$ ñtawata- „leader“). 
hiér. ROI-tis et lycien $\chi$ ñtawati, mais rien ne s'y oppose formellement “. ${ }^{62}$ Dieselbe Auflösung der logographischen Schreibung gibt R. WerNER. ${ }^{63}$ Wahrscheinlicher ist aber eine Auflösung der Schreibung als hassuuatti. ${ }^{64}$

\section{3. lykisch $\chi \tilde{n}$ tawati-}

Das Lykische weist das Lexem $\chi$ ñtawati- (F. STARKE: $\chi$ ñtawate $\left.(/ i){ }^{65}\right)$ auf, das zu der Wurzel $\chi$ ñtawa ,führen" gehört. In der lykisch-griechisch-aramäischen Trilingue von Xanthos ist $\chi$ ñtawati- mit griechisch $\beta \alpha \sigma \lambda \lambda \varepsilon v ́ \varsigma$ „König“ übersetzt. ${ }^{66}$ H. C. Melchert führt das Wort als $\chi$ ñtawat(o)- „,ruler, king“ ${ }^{67}$

\section{4. karisch}

Zugehörig ist auch karisch kסoúš (attributiv zu pismašk „Psammetich“) $<* /$ hạ $^{\text {n }}$ dauṣa/ < */handaưa-șa/ $<* /$ hantāūāa-dzāa / „Vorsteher, König“, dessen Bedeutung durch die 1996 gefundene griechisch-karische Bilingue von Kaunos deutlich wurde. ${ }^{68}$

$\mathrm{Zu}$ den nach / hantaw-a-d/ folgenden Hieroglyphen: $\mathrm{w}$ ist entweder als Konsonant /w/ oder Vokalschreibung [/u/bzw. /o/] zu verstehen; $\square \check{\text { s }}$ war im Mittleren Reich ein palatalisierter Frikativ $/ \int /$, dessen Korrelat vorher ägyptisch $\Pi / s /$ darstellte. ${ }^{69}$ Ich setze eine Notation des Nom. Sing. hantauxattis an (ein zunächst erwogener Nom. Plural wäre in diesem Fall (i-Stamm) formal mit dem

62 E. Laroche, L'inscription lycienne, in: H. Metzger, Fouilles de Xanthos VI: La stèle trilingue du Létôon, Paris 1979, 49-127: 105.

63 R. WERnER, Kleine Einführung ins Hieroglyphen-Luwische. Unter Mitarbeit von B. Lüscher, OBO 106, Freiburg Schweiz/Göttingen 1991, 92. Ohne Lesung bei P. MERIGGI, Hieroglyphen-hethitisches Glossar, Wiesbaden 1962, 173f.: LUGAL-ti(a)s-.

64 F. STARKe (brieflich, 12. 7. 2001).

65 Brieflich (12. 7. 2001).

66 E. Laroche, L'inscription lycienne, in: H. Metzger, Fouilles de Xanthos VI: La stèle trilingue du Létôon, Paris 1979, 49-127: 56, 64, 84, bes. 104-106; A. HeubECK,

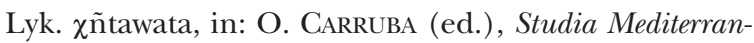
era 1, P. Meriggi dedicata, Pavia 1979, 247-259.

67 H. Craig Melchert, Lycian Lexicon, $2^{\text {nd }}$ fully rev. ed., Chapel Hill 1993, 92, dort auch $\chi$ ñtawa- ,to rule'; $\chi$ ñtawata- ,rule, kingship“, A. Gusmani, IF 68, 268ff.; GenAdj $\chi$ ñtawatehi.

68 I. HajnAL, ,Jungluwisches“ *s und die karische Evidenz, Kadmos 37 (1998), 80-108: 90f. (nach AdIEGO, Kadmos 34 (1995), 18-34: 19f.); ders., Die karisch-griechische Bilingue 44* aus Kaunos: ein erster Augenschein, Kad-

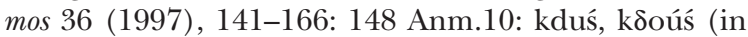
M 50 nominat. Regens; oder Verbalform /hantāuā-s/
Nom. Sing. identisch ${ }^{70}$ ), bei der in der vorliegenden Notation allerdings als Fehler $(/ \mathrm{u} /$ statt /i/) angesetzt werden müßte. Vielleicht kann aber auch eine Vorlage mit der Notation * $\square$ oder

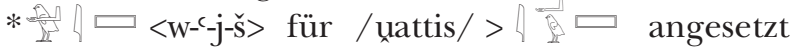
werden. Das unterschiedliche Determinativ des gefallenen Feindes (analog zu den Ächtungstexten) im Gegensatz zur sitzenden Person in den Vermerken $1 / 3$ erklärt sich am ehesten durch eine andere Vorlage. Damit läge im Vermerk der Sinuhe-Erzählung der chronologisch früheste explizite Beleg für luwische Fürstentümer vor, nachdem die luwische Besiedlung Südanatoliens nach 2000 abgeschlossen war (s. unten §5). Der folgende Ausdruck gibt nun offenbar das Territorium der Herrscher an. Traditionell wird 1 F.HзŚT, gelesen doch merkte schon Gardiner, der die autoritative Edition des Berliner Manuskripts B

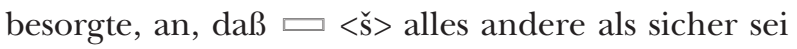
und eine andere Lesung korrekter sein könnte, als welche ich $\approx<\check{c}>$ vorschlage. ${ }^{71}$ Wenn wir hnt hier als „vorderer Landesteil, Süden“72 verstehen, heißt

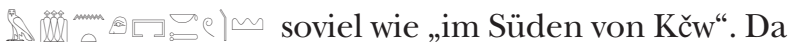
eines der Hauptsiedlungsgebiete der Luwier die Landschaft Kizzuwadna im Südosten Anatoliens war und anatolisches $<\mathrm{z}>$ der Affrikata /ts/ entspricht, die ägyptisch durch $\approx<<\check{c}>$ wiedergegeben wird, hatte ich zunächst in der Notation „er herrschte“?), 146: kdoús (mit berufsbezeichnender Endung); D. SCHÜRR, Kaunos in lykischen Inschriften, Kadmos 37 (1998), 143-162: 146f.: *kdusi „König“, belegt in Graffito in Abydos Ab. $29 \mathrm{~F}=6 \mathrm{Y}<$ *hantowassí:- ; adjektivisch auf Münchner Silberarmreifen, Abl. auch auf Stele von Hyllarima; aram. Umschrift kndws .

69 Ein Beleg dafür aus dem frühen Mittleren Reich dürfte der Frauenname $\sqrt{\square}$ k-b-š-t-j. $j_{2}=$ amurritisch kabśati „mein Lamm“ auf CG 20441 (K. LANGE, H. SCHÄFER, Grab- und Denksteine des Mittleren Reichs im Museum von Kairo, Le Caire 1902-1908, II, 39) sein.

70 Nach brieflicher Mitteilung von F. STARKE (12.7.2001). Nur bei der Annahme eines Plurals könnte diese Schreibung als Argument dafür dienen, daß die Pluralmorpheme des Luwischen erst nach 1900 v.Chr. vom Akkusativ aus neu gebildet wurden; zu letzterem s. F. STARKE, Die Kasusendungen der luwischen Sprachen, in: J. TISCHLER (Hg.), Serta Indogermanica. Festschrift für Günter Neumann zum 60. Geburtstag, Innsbrucker Beiträge zur Sprachwissenschaft, Bd. 40, Innsbruck 1982, 407-425.

71 S. Möller, Hieratische Paläographie, I, 528 (ev. über $w$ gesetzt?).

72 hnt „Anfang, Süden eines Landes“: Wb. III 306; C. VANDERSLEYEN, Les Guerres d'Amôsis, fondateur de la XVIII ${ }^{e}$ dynastie, Bruxelles 1971, 112. 
Teil des Toponyms Kizzuwadna vermutet. Nach einer Vermutung E. Laroches, ${ }^{73}$ der sich auch H. Eichner anschloß ${ }^{74}$ wäre nämlich in dem Element $u a d n$ - ein Wort für „Land“ zu sehen, so daß das Toponym Kizzuwadna etymologisch „Kizz-Land“ bedeuten würde. In diesem Fall wäre für die Schreibung des Sinuhe die Auslassung des Namenselementes „Land“ in der Umschrift erklärbar, sofern der etymologische Sinn des Toponyms als noch bekannt vorausgesetzt werden kann; umso mehr, als das hinzugesetzte generische ägyptische Determinativ ja anzeigte, daß es sich um ein fremdes Land handelte. Gegen diesen Ansatz sprechen verschiedene sprachliche Indizien, ${ }^{75}$ die einen hurritischen Ursprung des Namens Kizzuwadna befürworten; außerdem scheint die ältere, für die Zeit des Sinuhe in Frage kommende Bezeichnung der Landschaft Adana/Adanija gewesen zu sein, ${ }^{76}$ die ägyptisch als 14 I I $14 \sim$ belegt ist. ${ }^{77}$ Dagegen scheint eine andere Anschlußmöglichkeit vielversprechender, die ich F. STARKE verdanke. ${ }^{78}$ Ein weiterer luwischer Namei der Gegend Adana/Kizzuwadna war *Kau-/Kaua-, von dem hieroglyphen-luwisch im Karkamis des 10. Jh.s die Adjektivbildung kauxizza„kawäisch“ belegt ist. Zeitlich vor diesen Beleg (und die neuassyrischen Wiedergaben Qawe/Quwe) führen aus dem 2. Jahrtausend v. Chr. die Umschrift KUR Qa-a-ụ́[-e] in einem Brief Ramses' II. ${ }^{79}$ und die von E. EDEL nachgewiesene ägyptische Notation \& $\mathrm{H}$-w3 W3T. Wenn mit Edel die unterschiedliche Vertretung des velaren Anlauts dadurch zu erklären ist, daß er ein stark aspiriertes $\mathrm{k}$ war, ${ }^{80}$ könnte in der Tat kalligraphisch empfundenen Zeichenfolge

73 E. LARoche, in: H. MetzGer et al., La stèle trilingue du Létôon, Fouilles de Xanthos VI, Paris 1979, 67.

74 Schriftliche Mitteilung vom 8 Juni 2000.

75 F. STARKE, Untersuchung zur Stammbildung des keilschriftluwischen Nomens, StboT 31, Wiesbaden 1990, 468 Anm. 1705.

76 F. STARKe (brieflich am 12.7.2001), dem ich auch die Kenntnis der Arbeit von N. Oettinger, in: A. Hintze et al. (Hg.), Anusantatyai. Festschrift für Johanna Narten zum 70. Geburtstag, MSS Beiheft 19, 2000, 181-187, verdanke, die das von Laroche und Eichner beigezogene heth. udnē anders als „Flußtalgebiet" bestimmt.

77 E. EDEL, Neue Identifikationen topographischer Namen in den konventionellen Namenszusammenstellungen des Neuen Reiches, SAK 3 (1975), 49-73: 63. Diese Notation ist als Beleg für eine vergleichbare Aphärese der Anlautsilbe meinen Ausführungen zu äg. tl < hurr. adal (UF 31, 1999, 692) hinzuzufügen.

78 Schriftlich am 12. 7. 2001. ev. bei Umsetzung aus Kolumnenschreibung - oder einer Notation $\approx 0) \sim$ mit seitlich notiertem $<\mathrm{w}>\mathrm{zu}$ 巳e $\cong$; vgl. Anm. 71) für kaưizza- „kawäisches (Land)“ stehen. Die Verwendung adjektivischer Bildungen zur Bezeichnung von Gebieten ist für das Luwische charakteristisch. Unter Zugrundelegung der vorgeschlagenen Emendationen verstehe ich den zweiten Vermerk als:

„Der hantawattis (luwisch ,Herrscher') aus dem Süden von Kauxizza (dem Kawäischen Land)“

\section{§3.3 Der dritte Vermerk}

Der dritte Vermerk ist, was die Ortsangabe …를 matisch: „in den zwei Ländern der Fenchu“. Obwohl die Kommentatoren gemäß dem (seit der 1. Zwischenzeit) üblichen ägyptischen Sprachgebrauch meist einen Plural emendiert haben (vgl. oben die Übersetzungen [1], [2], [3], [4], [7], [8], [9], [10], [18], [19]; in einen Singular vereinfacht in [12], [13], [14], [16], [17]), möchte ich den Dual als lectio difficilior beibehalten ${ }^{81}$ (so in der Minderheit der Übersetzungen: [5], [11], [15]). Die Herrschaft über zwei Territorien ist auch im mesopotamischen Bereich häufig belegt. ${ }^{82}$ Der Begriff fnh.w deckt einen geographisch weiten Bereich Vorderasiens ab; die z.B. auf dem Streitwagen Thutmosis' IV. darunter subsummierten Gebiete umfassen Mesopotamien und Syrien. ${ }^{83}$ Etymologisch ist der Begriff fnh.w nicht geklärt. Die ursprünglich von K. SETHE vorgeschlagene (und u.a. von E. Edel übernommene ${ }^{84}$ ) Deutung als ägyptische Bezeichnung „Zimmerleute“ für die

79 E. EDEL, Die ägyptisch-hethitische Korrespondenz aus Boghazköi in babylonischer und hethitischer Sprache, Abhandlungen der Rheinisch-Westfälischen Akademie der Wissenschaften Bd. 77, Opladen 1994, II, 95, 120.

80 E. Edel (wie Anm. 79), $64 f$.

81 Gegen C. VANDERSLEYEN, Les guerres d'Ahmosis, fondateur de la XVIII e dynastie, Bruxelles 1971, 112 (,klarer Fehler $\left.{ }^{6}\right)$. Der Dual erscheint in ägyptischen Texten sonst erst in ptolemäischer Zeit ( $a a O$. 103f.), wo mit den beiden Ländern die phönizische Küste und Koile-Syrien gemeint zu sein scheint ( $a a O$. 118).

82 König von Sumer und Akkade; (der elamische) König von Susa und Anschan; der früheste bislang bekannte hurritische Herrscher Tiš-adal ist „König von Urkeš und Nagar".

83 VANDERSLEYEN (wie Anm. 81), $111 \mathrm{f}$.

84 E. EDEL, Die Ortsnamenlisten aus dem Totentempel Amenophis III (Bonner Biblische Beiträge 25), Bonn 1966, 30. Vgl. noch Green (wie Anm. 33), 40. 
„Holzfällerleute des Libanon“ scheint recht verwegen, eine hurritische Herleitung v.a. in chronologischer Hinsicht schwierig. ${ }^{85}$ Die Herrscherbezeichnung mit dem im Neuen Reich belegten geographischen Namen $\rightleftharpoons$ a zwischen Kftjw (Kreta) und Syrien erscheint, zusammengestellt und als „Minos“ identifiziert worden. Neben den oben bei der Kritik der These Kitchens vorgebrachten Argumenten wäre hier zu fragen, ob ein kretischer Personenname Minos in dieser Gestalt oder ein dem legendären Minos ursprünglich vielleicht zugrunde liegender minoischer Königstitel $^{87}$ zu Beginn des 2. Jahrtausends v. Chr. existierte. ${ }^{88}$ Eine plausible Deutung ist aber schwierig; um so mehr, als in diesem Fall Handschrift $B$ die auf den ersten Blick einzige Lesart bietet, während AOS stattdessen $h r$ rțj.t s.sm.w=k „beim Setzen deiner Verfügungen“ notiert, das vielleicht als Deutungsversuch einer nicht mehr verstandenen hieratischen Vorlage

${ }^{85}$ Man könnte die Adaptation eines hurritischen fabanahhe/fabanage „Bergbewohner" (s. I.M. Diakonoff, Hurrisch und Urarläisch (Münchener Studien zur Sprachwissenschaft 6), 1971, 71 Anm. 72; ägypt. <h > mit ursprünglichem Lautwert $/ \dot{\mathrm{g}} /$, bis MR $>/ \mathrm{h} /$ ) postulieren, das im Neuen Reich ägypt. $<$ pbh $>$ notiert wird (E. EDEL, Neue Identifikationen topographischer Namen in der konventionellen Namenszusammenstellung des Neuen Reiches, $S A K 3$, 1975, 49-73: 51-54). Der früheste ägyptische Beleg stammt aber schon aus der Region des Niuserre (um 2430 v. Chr.); der nächste aus der 6. Dynastie: J. LECLANT, T. P. Pépi Ier, VII: Une nouvelle mention des Fnhw dans les Textes des Pyramides, SAK 10 (FS W. Helck), 1984, 455-460. Der ägyptische Ausdruck dürfte daher zu früh belegt sein, um eine Übernahme der hurritischen Ableitung darstellen zu können. Zur Frühgeschichte der Hurriter s. unten Anm. 110.

${ }^{86} \mathrm{~J}$. Vercoutter, L' Egypte et le monde égéen préhellénique: élude crilique des sources égypliennes (du débul de la XVIII à la fin de la XIX $X^{e}$ dynastie), BdE 22, Le Caire 1956, 160ff.; EDEL (wie Anm. 84), 7

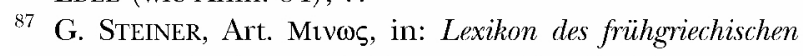
Epos, 15. Lieferung, Göttingen 1993, 224f. (ursprünglich vielleicht ,minoischer" Herrschertitel, etwa „(Priester) König").

${ }^{88} \mathrm{Zu}$ der Gestalt des Minos bzw. dem bronzezeitlichen Kreta s. R. HÄGG, N. MARINATOS (eds.), The Minoan Thalassocracy. Myth and reality. Stockholm/Göteborg 1984; P. REHAK (ed.), The Role of the Ruler in the Prehistoric Aegean, Aegaeum 11, Liège 1995; R. LAFFinEUR, W.D. Niemeier (eds.), Politeia. Society and State in the Aegean Bronze Age, Aegaeum 12, Liège 1995; M. Blomberg, G. Henriksson, Minos Enneoros. Archaeo- erklärt werden kann. Für die Interpretation der Notation sind folgende Punkte wichtig:

mn.w „Denkmal“) als $<$ mnw $>$ aufgefaßt werden;

ches zwar den Silbenwert /man $/,^{89}$ steht aber in dem einzigen verfügbaren Beleg des Mittleren Reiches vso. 11a) = munahhima für $/$ mun $/$ (oder omnivalent $/ \mathrm{mVn} /)^{90}$

- für oe kann vielleicht der Wert /nV/ angesetzt werden. $^{91}$

Als ursprüngliche Notation der Herrschaftsbezeichnung vermute ich * $\|^{\prime}$ haplographisch im Hieratischen nach ausgefallen (Hyperkorrektur für angebliches * l) wie direkt nach unserem Ausdruck eine Haplographie zu | statt 19 führte (s. \$4). Wird dieser Restitutionsvorschlag akzeptiert, schlage ich vor, hier <j>-mnnw-ŝ = *aa-múnə-nə-ś als approximative Wiedergabe astronomical Light on the Priestly Role of the King in Crete, in: P. Heliström, B. Alroth (eds.), Religion and Power in the Ancienl Greek World. Proceedings of the Uppsala Symposium 1993, Uppsala/Stockholm 1996, 27-39. Abwegig scheint mir der etymologische Versuch Y. Arbeitmans, Minos, the „Oaristes“ of Great Zeus: á-, á-, and o-copulative, the Knossan royal titulary and the Hellenization of Crete, in: Yokl L. Arbeitman (ed.), A Linguistic Happening in Memory of Ben Schwartz. Studies in Anatolian, Italic, and other Indo-European Languages, Louvain-la-Neuve 1988, 411-462, S. 437: „In the three present examples, Balto-Slavic mil-, Hieroglyphic Luwian mit, and Cretan (an IE stratum at least, and one which left some anthroponyms and toponyms, and the titulary, i.e. it need not be the language of the people of Crete) $m i n$-, I rather think that we are dealing with a phenomenon different from IE stem building. In a word, varied formants of a past passive participle, at least in origin, i.e. mit-, mil-, and min-, are all three IE expressions of a state: ,endowed with (the) benignity (of/from the deity)', hence ,beloved, servant/worshipper", etc.; more simply and less literally, ,Covenant Partner" (of the deity)."

${ }^{89}$ J. E. HосH, Semitic Words in Lgyptian Texts of the New Kingdom and Third Intermediate Period, Princeton 1995, 508.

90 T. SCHNEIDER, Die semitischen und ägyptischen Namen der syrischen Sklaven des Papyrus Brooklyn 35.1446 verso, UF 19 (1987), 255-282: 261f. (Deutung zuerst von W. F. Albright); vgl. M. P. STReck, Das amurritische Onomastikon der altbabylonischen Zeit. Bd. 1: Die Amurriter, die onomastische Forschung, Orthographie und Phonologie, Nominalmorphologie, AOAT 271, Münster 2000, 339 (muQaTTiL Part. des D-Stammes). 
von hurritisch amummi $=$ ne $=\check{\text { s }} /$ amummines $/$ (Ergativ Sg.) „Hoheit, Souverän“ zu betrachten. Dieser Ansatz ist zwar nicht derart überzeugend wie die für die Einträge 1/2 vorgeschlagene Interpretation; ich sehe aber gegenwärtig keine andere Lösung.

Das zugrundeliegende Wort ist amumi /amum$\mathrm{mi} /$, das gewöhnlich von amumi /amomi/ „Botschaft" getrennt wird, ${ }^{92}$ mit den abgeleiteten Berufstermini amumm-i=nni (in der hurritischhethitischen Bilingue aus Bogazköy durch hethitisch manijaḩhiaš išhaš „Verwalter“ wiedergegeben ${ }^{93}$ ), amumm-i=kk-onni und amummi=hhuri „Verwalter“. ${ }^{94}$ Als Grundbedeutung ist „Hoheit, Souverän“ plausibel, da in einem dreisprachigen Vokabular aus Ugarit die Adjektivableitung amumiašše mit MAH = șîru „erhaben, erstrangig“ (d.h. hoheitsvoll) übersetzt wird ${ }^{95}$ Der dritte Vermerk kann daher mit Vorbehalt verstanden werden als:

„Der <’a>múnənəš ( hurritisch amummines ,Souverän') in den zwei Ländern der Fenchu“"

\section{§4 Der Rahmentext}

Die drei Herrschervermerke sind in den Text eingebettet durch eine Einleitung und Ausleitung von ebenfalls noch sehr unterschiedlich verstandener Aussage. In der einleitenden Formulierung wc̣̆ MÇ̌sT grt hm $=k$ rț.t jnt $=f$ (dann die drei erörterten Vermerke) stehen korrekt zwei Verben des Veranlassens (wč, rțj), die auf eine mittelbare Anweisung (,zu veranlassen befehlen“) deuten. Die Mehrzahl der Übersetzer haben diesen Textbefund im Sinne eines unmittelbaren Veranlassens vereinfacht. Korrekt sind in dieser Hinsicht von den zu Beginn zitierten Übersetzungen des Passus nur folgende sieben:

[2] „May thy Majesty command to cause to be brought Maki from Kedme, Khentiaush from Khentkesh, Menus from the lands of the Fenkhu" (GARDINER 1916) ${ }^{96}$

[6] „Que ta Majesté ordonne de faire amener [les trois princes asiatiques]" (POSENER 1956) ${ }^{97}$

[10] „May then your Majesty command to have brought to you the prince of Meki from Qedem, the mountain chiefs from Keshu, and the prince of Menus from the lands of the Fenkhu." (Lichtheim 1973) ${ }^{98}$

[12] „Auch möge deine Majestät befehlen, daß man veranlasse, den Meki zu holen aus dem Ostland, den ChentiJausch aus Chentikesch und den Menus aus dem Lande der Phönizier." (SCHÜSSLER 1980) ${ }^{99}$

[13] „Deine Majestät möge ferner befehlen, daß man Meki aus Kedem, Chentijawasch aus Chent-kuschu und Menus aus dem Land der Phönizier holen lasse." (BLuMENTHAL 1982) ${ }^{100}$

[16] „Deine Majestät möge aber befehlen, daß man holen lasse Meki aus Kedem, Chenti-jawasch aus Chent-kuschu, Menus aus dem Land der Phönizier." (Blumenthal $1995)^{101}$

[18] „Now, may your Majesty command that he be made to bring the Meki man from Qedem, the settler from out of Keshu, and the Menus man from the lands of the Fenkhu." (PARKInSON 1997) ${ }^{102}$

Sämtliche Interpreten der Notiz gingen bisher davon aus, daß die registrierten Herrscher nach Ägypten geholt oder gebracht werden sollen. Zuletzt vermutete C. Obsomer, Sinuhe habe die Fürsten vielleicht auf seinem Rückweg zur Residenz von Itjtaui mitnehmen können. ${ }^{103}$ Zwar ist die Vorladung levantinischer Fürsten - so im Fall des nach

91 J. ZeIdLER, A New Approach to the Late Egyptian „Syllabic Orthography“, in: Atti Sesto Congresso Internazionale di Egittologia. Volume II, Torino 1993, 579; vgl. E. EDEL (wie Anm. 84), 63.

92 I. WEGNER, Einführung in die hurritische Sprache [Umschlag: Hurritisch. Eine Einführung], Wiesbaden 2000, 202.

93 E. NEU, Das hurritische Epos der Freilassung I. Untersuchungen zu einem hurritisch-hethitischen Textensemble aus Hattuša, StBoT H. 32, Wiesbaden 1996, 84f.171; G. WILHELM, Or 61 (1992), 129; Jean Catsanicos, L'apport de la bilingue de Hattuša à la lexicologie hourrite, in: JEANMarie Dunand (ed.): Amurru 1: Mari, Ébla et les Hourrites. Dix ans de travaux. Première partie. Actes du colloque international (Paris, mai 1993), Paris 1996, 252; M. GIORGERI, Schizzo grammaticale della lingua Hurrica, La Parola del Passato 55 (2000), 171-277:193, 197, 211 (zu am = „beobachten“).

94 I. WEGNER, Einführung in die hurritische Sprache, Wiesbaden 2000, 49, 51 .

95 B. André-SALVInI/M. SALVInI, Un nouveau vocabulaire trilingue sumérien-akkadien-hourrite de Ras Shamra, SCCNH 9 (1998), 3-40: V 9'; p. 9.

96 A. H. Gardiner, Notes on the Story of Sinuhe, Paris 1916, 84.

97 G. Posener, Littérature et politique dans l'Egypte de la XII dynastie, Paris 1956, 113.

98 M. Lichtheim, Ancient Egyptian Literature. A Book of Readings. Vol. I: The Old and Middle Kingdoms, Berkeley/Los Angeles/London 1973, 230.

99 K. SCHÜSSLER, Märchen und Erzählungen der Alten Ägypter, $1980,84$.

${ }^{100}$ E. Blumenthal (Hg.), Altägyptische Reiseerzählungen. Die Lebensgeschichte des Sinuhe - Der Reisebericht des WenAmun, Leipzig 1982, 18.

${ }^{101}$ E. Blumenthal, Die Erzählung des Sinuhe, TUAT III: Weisheit, Mythen und Epen III, Gütersloh 1995, 904.

${ }^{102}$ R. B. PARKInson, The Tale of Sinuhe and other Ancient Egyptian Poems 1940-1640 BC, Oxford 1997, 38.

103 C. Obsomer (wie Anm. 4). 
Achetaton zitierten Aziru von Amurru - oder die gewaltsame Überstellung vermutlich illoyaler Regenten - so im Fall des in einem Käfig gefangenen Fürsten der Amarnazeit ${ }^{104}$ - in der Tat bezeugt. Die vorgeschlagene Neudeutung macht aber ein Verständnis, nach dem Herrscher syrisch-südostanatolischer Territorien persönlich nach Ägypten hätten gebracht werden sollen, kaum plausibel. Eine Lösung eröffnet sich hier durch ein anderes syntaktisches Verständnis. jnt=f ist zwar als Subjunktiv „daß er bringe" nach rț.t aufzufassen; die drei Herrschervermerke sind aber nicht das Objekt zu jnt=f, sondern zu rțj.t. Dieses Objekt ist nun aber überlang und wird selber nochmals durch den erklärenden $p w$-Satz mit nachfolgender Partizipialfügung haj.w pw mtr.w rn.w hpr.w m mr.wt=k gestreckt. Eine reguläre Syntax * wç̣grt ḥm =k rṭj.t Objekt [die drei Herrschervermerke + Erklärung] jnt=f „Möge nun deine Majestät befehlen, die Herrscuier zu veranlassen, daß er (Geschenke) überbringt" ist dadurch verunmöglicht, so daß der subjunktivische Objektsatz vor das direkte Objekt vorgezogen wird („Möge nun deine Majestät befehlen anzuweisen /daß er (Geschenke) überbringt/ die HerRscher“; „Daß er (Geschenke) überbringt, befehle nun deine Majestät zu veranlassen die Herrscher“). Werden die Herrschertitel so neu als Objekt des $r t j$, nicht des jnj aufgefaßt, erscheint Sinuhe als Mittelsmann und Überbringer diplomatischer Geschenke dieser Regenten an den ägyptischen Hof. Dabei verstehe ich jnt=f als elliptische Formulierung für jnt=f jn.w, so wie jnj + Landesname „ein Land bringen“ als elliptischer Ausdruck für „die Produkte/Gaben eines Landes bringen“ bezeugt ist. ${ }^{105}$ Anders als traditionell üblich möchte ich auch den erklärenden Abschluß der Herrschernotiz He per verstehen. Zwar hat man für den Ausdruck $\mathrm{mtr}$ rn auf ein Beamtenepitheton des Mittleren Reiches und eine Stelle der Armant-Inschrift Thutmosis' III. verwiesen ${ }^{106}$ und häufig an Herrscher mit „gut beleumundeten Namen" oder guter Reputation

${ }^{104}$ M. Abdul-Kader Mohımmad, The Administration of Syro-Palestine during the New Kingdom, ASAE 56 (1959), 105-137, pl. I.

${ }^{105}$ S. AnnLex I 77.0317.

${ }^{106}$ Hinweis auf das Beamtenepitheton auf BM 572 bei Gardiner (wie Anm. 8); E. Blumenthal, Untersuchungen zum ägyptischen Königtum des Mittleren Reiches, Berlin 1970, 238 (F 3.31) (beurteilt das Vorkommen in der gedacht (s. die zu Beginn aufgelisteten Übersetzungen). Verstehen wir, wie oben vorgeschlagen, $r n$ im Sinne von „(Herrschafts-)Titel“, könnte eher an Herrscher, deren Titel bezeugt (im Sinne von: diplomatisch beglaubigt) sind, gedacht werden. Aber auch diese Interpretation mißachtet die Personendeterminierung, die mtr.w deutlich als „Zeugen, Gewährsmänner“ ausweist. Ich verstehe ḥı.w pw mtr.w rn.w deshalb als „Die Gewährsmänner der Titel sind Herrscher" - konkrete Träger der zitierten Herrschertitel sind es, die für ihre Korrektheit bürgen.

Abschließend ergibt sich als hypothetisches Gesamtverständnis der Herrschernotiz des Sinuhe (B 219-222):

Daß er (Tribut) überbringt, möge nun deine Majestät befehlen $\mathrm{zu}$ veranlassen

den Mkj< $<$ m $>\approx$ Mēkim (semit. ,König“; falls < malki/um) in Qṭwm $\approx$ Qațanum,

den hntwj'wš $\approx$ Hantawattis (luw. "Herrscher") aus dem Süden von Kwč $\approx$ Kaưizza (dem Kawäischen Land),

den $<\mathbf{j}>$ mnnwś $\approx$ Amummines (hurr. ,Souverän“) in den zwei Ländern der Fenchu.

Die Gewährsmänner der Titel sind Herrscher, die in Loyalität zu dir existieren.

\section{\$5 Historische UND LiteraRISCHE KonseQUenZeN}

Der Befund der Textüberlieferung des Sinuhe erlaubt keine wesentlich spätere Entstehungszeit als 1900-1850 v. Chr. (Regierungen Sesostris' I. oder Amenemhats II.). Die in der Diskussion der Einzelvermerke festgestellten Divergenzen bzw. Unstimmigkeiten der Herrschernotiz könnten allerdings darauf hinweisen, daß sie nicht erst bei der Abschrift aus einer Vorlage in Hs. B entstanden sind, sondern der Autor des Sinuhe selber nicht mehr präzis über den Sinn und den korrekten fremdsprachigen Wortlaut im Bilde war. Es wäre dann denkbar, daß die Herrschernotiz des Sinuhe aus Aktenvermerken, die beispielsweise in der Kanz-
Herrschernotiz des Sinuhe als Übertragung einer Beamtentugend auf Ausländer); D. M. Doxey, Egyptian Non-Royal Epithets in the Middle Kingdom: a Social and Historical Analysis, PÄ 12, Leiden 1998, 316. Zum Passus der Armant-Stele (Urk. IV 1246, 9), der auch von der Loyalität ausländischer Herrscher handelt, BARNs (wie Anm. 6), 24. 
lei der Residenz von Itjtaui vorlagen, in die Urfassung der Erzählung übernommen wurden. ${ }^{107}$

Aus der vorgeschlagenen Neuinterpretation der Herrschernotiz der Sinuhe-Erzählung ergeben sich Konsequenzen für drei Bereiche: 1. die politische Geschichte Nordsyriens und Anatoliens um 1900 v. Chr.; 2. die Frage nach dem Sinn der SinuheErzählung; und 3. das Verständnis politischer Beziehungen und Diplomatie im frühen 2. Jt. v. Chr.

1. Der Passus vermittelt überaus wichtige Informationen zur politischen Geographie und Geschichte Nordsyriens und Südostanatoliens zu einer Zeit, in der andere Quellen kaum oder nur unzureichend verfügbar sind. Das nach unserem Verständnis zunächst genannte Reich von Qațanum schließt nördlich an das palästinische Gebiet (Retjenu) an, dessen Loyalität zu Ägypten der Rahmenvermerk konstatiert. Während die Archive von Mari und Alalach für die zwei Jahrhunderte zwischen 1800 und 1600 v. Chr. Qațanum als zentrale Macht Syriens charakterisieren, ist die politische Situation hier hundert Jahre früher nicht genauer faßbar. Der Eintrag des Sinuhe bezeugt, daß schon zu dieser Zeit das Reich von Qațanum als maßgebende politische Instanz Syriens betrachtet wurde. Von überragender Bedeutung ist jedoch der zweite Eintrag, der einen luwischen Herrschertitel für das Gebiet Kauxa (Kilikien) verzeichnet, bei dem es sich um den frühesten für Anatolien belegten indoeuropäischen Herrschertitel handeln würde und einen expliziten Nachweis von politischen Strukturen der neuen luwischen Bevölkerung zu Beginn des 2. Jt.s v. Chr. ${ }^{108}$ Zwar zeichnen auch die Doku- mente der altassyrischen Handelskolonien das Bild eines in viele voneinander unabhängige und sich befehdende Fürstentümer zersplitterten Territoriums. Ihre Fürsten werden in den altassyrischen Texten aber akkadisch als rubā'u bezeichnet, was uns über ihre sprachliche oder ethnische Zugehörigkeit im dunkeln läßt. Es besteht heute Einigkeit darin, daß die indoeuropäische Besiedlung Anatoliens schon nach 2300 v. Chr. erfolgt sein muß, da anders die erhebliche sprachliche Auseinanderentwicklung des Hethitischen und Luwischen, wie sie schon in den ersten überlieferten Schriftzeugnissen offensichtlich ist, nicht erklärt werden kann; ${ }^{109}$ die luwische Besiedlung ist im 19. Jahrhundert mit Sicherheit abgeschlossen. Der (in seiner Interpretation weniger gesicherte) dritte Vermerk bezeugt die zusätzliche politische Präsenz der Hurriter, einer seit dem letzten Viertel des 3. Jahrtausends nach Obermesopotamien, Nordsyrien und Ostanatolien einwandernden Bevölkerung, ${ }^{110}$ möglicherweise eher in dem östlich an das Territorium der Einträge 1/2 anschließenden Gebiet. Die Passage spiegelt somit schon für die Zeit um 1900 jene sprachlich und kulturell hochkomplexe Situation wider, wie sie aus späteren Jahrhunderten bekannt ist.

2. E. Blumenthal urteilte über die Erzählung des Sinuhe: „Der historische Informationswert der Dichtung sollte daher nicht überschätzt werden. Sie löst weder die Probleme um die Koregenz von Amenemhet I. und Sesostris I. und um die mutmaßliche Ermordung Amenemhets I., noch entwirft sie ein vorderasiatisches Itinerar, ein verläßliches Bild der
${ }^{107}$ Diese zeitliche Differenz könnte die unterschiedliche Verwendung des $\left.<^{c}\right\rangle=/$ d/ in der Wiedergabe des Titels hantawatti; vermutlich $=/ \%$ in der Umschrift des Gottesnamens 'ammu im Eigennamen 'mmwnnšj erklären, falls die bisherigen Deutungen akzeptiert werden. Zu letzterem Namen s. zuletzt L. D. Morenz (wie Anm. 4) 4f.; A. Loprieno, in: Stationen. FS R. Stadelmann, 216 n.41.

${ }^{108}$ F. STARKE (brieflich am 12.7.01) weist mich auf ein weiteres frühes Staatswesen vor der hethitischen Reichsbildung unter Anitta hin, das durch den Palast von Beycesultan im oberen Maiandros-Tal für die Zeit von 1900-1750 v. Chr. bezeugt ist.

${ }^{109}$ O. Carruba, Luwier in Kappadokien, in: D. Charpin, F. JOANnÈs (eds.), La circulation des biens, des personnes et des idées dans le Proche-Orient Ancien, Paris 1992, 251-257: 253 (schon um 1850 v. Chr. völlig durchgeführte phonetische Entwicklung des Luwischen), 254 n.4 (Besiedlung zw. 2300 und 2000; luwische Besiedlung im 19. Jh. v. Chr. schon abgeschlossen). Vgl. auch das Ethnikon nuwa'um/luwa'um in altassyrischen Urkunden und frühe luwische Elemente in Personennamen: P. Garelli, Les Assyriens en Cappadoce, Paris 1963, 139f.; A. M. JASINK, Hittite and Assyrian Routes to Cilicia, in: De Anatolia Antiqua / Eski Anadolu: travaux et recherches de l'Institut français d'études anatoliennes, I, Paris 1991, 253ff.

${ }^{110}$ P. Steinkeller, The Historical Background of Urkesh and the Hurrian Beginnings in Northern Mesopotamia, in: G. Buccellati, M. Kelly-Buccellati (eds.), Urkesh and the Hurrians, Malibu 1998, 75-98; M. SAlvINI, The Earliest Evidence of the Hurrians Before the Formation of the Reign of Mittanni, aaO., 99-115. Vgl. noch A. KunrT, The Ancient Near East, c. 3000-330 BC, 2 vols., London/New York 1995, 288: „It is, therefore, likely that the Hurrians were a cultural-linguistic group either having immigrated from the far Northeast or always located among the foothills and mountains fringing the northern Mesopotamiam and Syrian plains." 
Verhältnisse in Palästina-Syrien und der Beziehungen zwischen Ägypten und dieser Region. “111 Dieser Einschätzung muß, was die vorderasiatischen Informationen betrifft, nach der hier vorgelegten Interpretation des Abschnitts B219-222 widersprochen werden, der geradezu bemerkenswerte Fakten überliefert. ${ }^{112}$ Darüberhinaus scheint eine Klassifizierung des Sinuhe als entweder Reportage oder Fiktion, als entweder historisch oder ahistorisch, zu simplifizierend. Zwar präsentiert der Autor Sinuhe in einem fiktionalen Rahmen, einem als solchem unhistorischen Lebenslauf, der allerdings auf einer korrekten Faktenlage aufbaut. In dem konkreten Zweck, für den die Erzählung verfaßt wurde, ist sie aber ganz sicher auch als historische Quelle zu bewerten - sei dieser Zweck nun die Definition kultureller Ägyptizität ${ }^{13}$ oder ein spezifischerer politischer Inhalt. ${ }^{114}$ Zuletzt hat wiederum C. Obsomer die Vermutung befürwortet, die Erzählung sei für ägyptische Flüchtlinge im Ausland redigiert worden, die unter der Bedingung, daß sie Sesostris I. gegenüber loyal wären, nach Ägypten hätten zurückkehren können. ${ }^{115}$ Dagegen hat jüngst S. Morschauser die Sinuhe-Erzählung als Porträt eines „Knight Errant“ auf der Suche nach einem Herrn betrachtet, ${ }^{116}$ in dem auch das Motiv der Flucht seine sachliche Begründung habe. ${ }^{117}$ Loyalität ist sicher ein Hauptanliegen der Erzählung wie ebenso zweier weiterer politischer Schriften des frühen Mittleren Reiches, der Lehre für Amenemhet und der Loyalistischen Lehre. Zu der Stellung Sinuhes als Gefolgsmann (Leibwächter), d.h. Person in persönlicher und beruflicher Loyalität zu dem ägyptischen König korrespondiert zunächst die Aufnahme im palä-

111 Blumenthal, TUAT III, 886.

112 Vgl. auch A. F. RAINEY, The World of Sinuhe, IOS 2 (1972) 367-408; L. D. Morenz (wie Anm. 4).

113 A. Loprieno, Topos und Mimesis, ÄAT 48, Wiesbaden 1988, Kap. 5; G. Moers, Travel as Narrative in Egyptian Literature, in: Lingua Aegyptia. Studia monographica 2: Definitely: Egyptian Literature, Göttingen 1999, 43-61: 53; ders., „Bei mir wird es Dir gut ergehen, denn Du wirst die Sprache Ägyptens hören!“ Verschieden und doch gleich: Sprache als identitätsrelevanter Faktor im pharaonischen Ägypten, in: U.-C. SANDER, F. PAul, Muster und Funktionen kultureller Selbst- und Fremdwahrnehmung. Beiträge zur internationalen Geschichte der sprachlichen und literarischen Emanzipation, 2000, 45-99 (die Kenntnis dieses Beitrages verdanke ich G. Moers). Dieses Anliegen allein kann aber den Aufstieg des Sinuhe von der einfachen Position eines šmśw zu der eines Fürsten, die wiederum Bedingung des Sinuhe letztlich zuerkannten Begräbnisses ist, nicht erklären. stinischen Exil bei dem Herrscher Ammunenschi, der das Thema Loyalität aufgreift. Im Lichte obiger Interpretation von $m=$ Titel könnte hier auch Sinuhes Forderung an den palästinischen Oberherrn zu verstehen sein: „Schick zu ihm! Laß ihn deinen ,Namen“ (Königstitel als Definition der politischen Hierarchie) wissen! Äußere keinen Fluch gegen seine Majestät. Er (der König) wird dir gegenüber handeln, wie sein Vater zu tun pflegte. Er wird nicht versäumen, Gutes für ein Fremdland zu tun, das ihm loyal sein wird“. Mit dem Aufstieg des Sinuhe entfaltet die Erzählung eine zuletzt von C. Obsomer beschriebene weitgehende Analogie zwischen Sesostris und Sinuhe, ${ }^{118}$ die die Gefährdung von Macht und die Bedeutung von Loyalität herausstellt. Entscheidender noch, und m.W. bisher wenig beachtet, ist das Bild, das die Erzählung nach der Einsetzung Sinuhes als Herrscher eines bedeutenden Stammes durch seinen Patron Ammunenschi entwirft: Sinuhe wird nun bis zu seiner Rückkehr nach Ägypten de facto als palästinischer König oder Fürst beschrieben - er erhält eine neue Identität, hat sein Hofpersonal, das für ihn jagt und ihn bedient; er vermehrt und sorgt für den Ertrag des Landes; sein Hof ist die Drehscheibe des diplomatischen Verkehrs zwischen Nord und Süd. Sinuhe berät andere Stämme strategisch, er ist der wohltätige orientalische Herrscher, der dem Durstigen Wasser gibt, den Verwirrten auf den Weg setzt, sich des Beraubten annimmt, und gleichzeitig der Kriegsherr und Eroberer, der jedes Fremdland, gegen das er zieht, besiegt.

Indem Sesostris von sich aus Sinuhe Geschenke schickt und so dessen Herz erfreut „wie das des

${ }^{114}$ Vgl. zur Frage politischer Literatur jetzt noch J. AssMANN et al. (Hgg.), Literatur und Politik im pharaonischen und ptolemäischen Ägypten. Vorträge der Tagung zum Gedenken an Georges Posener, 5.-10. September 1996 in Leipzig, BdE 127, Le Caire 1999.

115 C. Obsomer, Sinouhé l'Egyptien et les raisons de son exil, Le Muséon 112 (1999), 207-271.

116 S. Morschauer, What Made Sinuhe Run: Sinuhe's Reasoned Flight, JARCE 37 (2000), 187-198. Vgl. zur Fluchtthematik jetzt noch D. C. SNell, Flight and freedom in the ancient Near East, Culture and History of the Ancient Near East 8, Leiden 2001.

${ }^{117}$ Es sei bei der Gelegenheit auf die interessanten inhaltlichen Übereinstimmungen zwischen Sinuhe und der Biographie des Idrimi von Alalach hingewiesen, denen einmal nachgegangen werden müßte. Vgl. KuHRT (wie Anm. 110), 289ff.

118 Dazu ausführlich Obsomer (wie Anm. 115). 
Herrschers von jeglichem (anderen) Fremdland“, stellt er diplomatischen Kontakt her mit einem palästinischen Fürsten, als welcher Sinuhe porträtiert ist. Da dieser palästinische Fürst Sinuhe sich in seiner Antwort an den König weiterhin als „Diener des Palastes“ definiert (dies rechtfertigt auch die anfangs vorgenommene Einstufung seiner Antwort als Immediatbericht) und seine gleichzeitige gesellschaftliche Einbindung in Retjenu und Anerkennung in Ägypten betont, wird hier offensichtlich das Idealbild des dem ägyptischen König loyalen palästinischen Klientelfürsten oder Provinzgouverneurs gezeichnet, wie es im Neuen Reich die Amarnabriefe herausstellen. Entwickelt die Sinuhe-Erzählung auch ein Modell außenpolitischer Loyalität zum ägyptischen König?

3. Die primäre oder sekundäre Bezeugung altorientalischer Herrschertitel im Sinuhe wirft im Verbund mit Funden wie insbesondere der AnnalenInschrift Amenemhats II. aus Mitrahine, ${ }^{119}$ die ein expansives militärisches Vorgehen des Mittleren Reiches im syrisch-südostanatolisch-zypriotischen Raum belegt, daran anknüpfend die Frage auf, ob nicht die Syrienpolitik des Mittleren Reiches weitaus intensiver aussah als bisher vermutet. So hatte etwa 1984 Dietrich Wildung formuliert: „Gerade angesichts der intensiven Südpolitik ist die Zaghaftigkeit des ägyptischen Ausgreifens nach Norden um so erstaunlicher". ${ }^{120}$ Geradezu eine Illustration der hier vorgeschlagenen Deutung, wonach die syrischanatolischen Herrscher dem ägyptischen König Tribute oder Geschenke zukommen lassen sollen, liefern die genannte Annalen-Inschrift Amenemhats II., die Tribut, den „die Kinder Asiens“ bringen, und Tribut aus einer Stadt Samparu (o.ä.) erwähnen, aber auch der Schatz von Tôd ${ }^{121}$ aus derselben Regierungszeit. ${ }^{122}$

Die Neudeutung der Passage führt außerdem zu der Feststellung, daß die Erzählung genau jene geopolitische und diplomatische Zweiteilung des Vorderen Orients skizziert, die auch die Amarna-Korrespondenz zeichnet: ein engerer ägyptischer Einflußbereich (die Klientelkönige Palästinas, hier die Gestalt des Ammunenschi) zum einen, ein internationaler Bereich (im Neuen Reich das hethitische Reich, Babylonien, Assyrien, Mitanni, Arzawa, Zypern; hier die in B219-222 vermerkten Herrscher), für den andere diplomatische Gepflogenheiten gelten. Ob für den näheren palästinischen Bereich im Mittleren Reich schon Ansätze einer eigentlichen Verwaltung bestanden, bleibe hier dahingestellt. ${ }^{123}$ Deutlich propagiert Sinuhe als ägyptischer Vertreter im Ausland ${ }^{124}$ aber loyale Beziehungen und diplomatische Kontakte. Damit stellt sich die Frage, ob und wie intensiv ein übergreifendes System von „Prestige and Interest" ${ }^{\text {"125 }}$ schon im Vorderen Orient des frühen Mittleren Reiches existierte.
119 A. Altenmüller, A. M. Moussa, Die Inschrift Amenemhets II. aus dem Ptah-Tempel von Memphis. Vorbericht, SAK 18 (1991), 1-48; C. EDER (wie Anm. 34), 176-195.

${ }^{120}$ D. WILdung, Sesostris und Amenemhat. Ägypten im Mittleren Reich, Fribourg/München 1984, 188.

${ }^{121}$ Kretische Silber- und Goldgefäße sowie Rollsiegel der mesopotamischen 3. Dynastie von Ur in Behältern mit dem Namen Amenemhats II. aus dem Gründungsdepot des Tempels von Tôd. Vgl. noch K. R. MaxwelLHyslop, A Note on the Anatolian Connections of the Tôd Treasure, Anatolian Studies 45 (1995), 243-250.

${ }^{122}$ Umgekehrt fand sich in Qatna ein Sphinx von Amenemhats II. Tochter Ita, der aber auch in späterer Zeit hierhin gelangt sein kann.

${ }^{123}$ Vgl. hier v.a. die z.T. im Sinne einer derartigen Verwaltung gedeuteten Funde aus Megiddo (Statuettenfragmente des Gaufürsten Thothotep II., Siegel eines Viehzählers) und weitere Hortfunde aus Palästina
(Information R. Schulz). Schließlich legt die Sinuheerzählung Sinuhe den Titel „Verwalter der Provinzen der Majestät in den Ländern der Asiaten“ bei. Bei diesem Titel soll es sich um einen fiktiven, da sonst nicht belegten Titel handeln. Vielleicht handelt es sich um einen programmatischen Titel, der dem ägyptischen König (Amenemhat II.?) die Option auf eine Verwaltung Palästinas eröffnen soll ?

${ }^{124}$ Vgl. noch für das Neue Reich E. B. Pusch, Ein Reisender in ferne Länder, in: M. SCHAdE-Busch (ed.), Wege öffnen. FS für R. Gundlach, ÄAT 35, Wiesbaden1996, 187-196; H. EL-SAADY, The External Royal Envoys of the Ramessides: a Study on the Egyptian Diplomats, MDAIK 55 (1999), 411-425.

125 M. LIVERANI, Prestige and Interest: International Relations in the Near East, ca. 1600-1100 B.C., Padova 1990; R. Cohen, R. Westbrook, Amarna Diplomacy. The Beginnings of International Relations, Baltimore/London 2000, $10 f$. 